\title{
Massless sunset diagrams in finite asymmetric volumes
}

\author{
F. Niedermayer ${ }^{a}$ and P. Weisz ${ }^{b}$ \\ ${ }^{a}$ Albert Einstein Center for Fundamental Physics, \\ Institute for Theoretical Physics, University of Bern, \\ Sidlerstrasse 5, 3012 Bern, Switzerland \\ ${ }^{b}$ Max-Planck-Institut für Physik, \\ 80805 Munich, Germany \\ E-mail: niedermayer@itp.unibe.ch, pew@mpp.mpg.de
}

\begin{abstract}
This paper discusses the methods and the results used in an accompanying paper describing the matching of effective chiral Lagrangians in dimensional and lattice regularizations. We present methods to compute 2-loop massless sunset diagrams in finite asymmetric volumes in the framework of these regularizations. We also consider 1-loop sums in both regularizations, extending the results of Hasenfratz and Leutwyler for the case of dimensional regularization and we introduce a new method to calculate precisely the expansion coefficients of the 1-loop lattice sums.
\end{abstract}

Keywords: Effective field theories, Sigma Models, Lattice Quantum Field Theory

ArXIV EPRINT: 1602.03159v1 


\section{Contents}

1 Introduction 1

2 Dimensional regularization at finite volume $\quad 2$

2.1 Massive propagator sums in DR 2

2.1.1 Sums with extra factor in the numerator 6

2.1.2 Keeping first order $D-d$ terms in $G_{r, 1} \quad 8$

3 Massless propagator sums in dimensional regularization $\quad 9$

$\begin{array}{ll}3.1 \text { The free propagator with periodic bc in DR } & 12\end{array}$

3.1.1 Propagator in infinite volume, $D$-dimensions 12

3.1.2 Calculating $g(x)$ for general $D \quad 13$

3.2 Evaluation of some 1-loop integrals using the representation in subsection 3.115

$\begin{array}{lll}3.2 .1 & \int_{x}[G(x)]^{2} & 16\end{array}$

$\begin{array}{lll}3.2 .2 & \int_{x} G(x) \ddot{G}(x) & 18\end{array}$

4 Massless sunset diagram with DR $\quad 19$

$\begin{array}{lll}4.1 & \Psi_{1} & 20\end{array}$

$\begin{array}{lll}4.2 & \Psi_{2} & 20\end{array}$

$\begin{array}{lll}4.3 & \Psi_{3} & 21\end{array}$

$\begin{array}{llll}4.4 & \Psi_{4} & 21\end{array}$

$\begin{array}{llll}4.5 & \Psi_{5} & 22\end{array}$

$4.6 \Psi_{6}$ and $\Psi_{7} \quad 23$

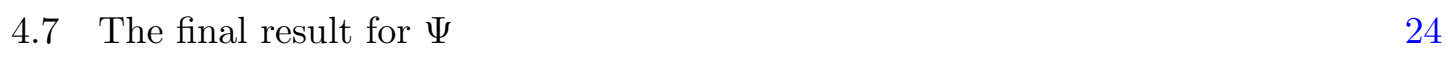

4.8 Checks 24

4.9 Other results 25

5 Dimensionally regularized integrals on a strip 26

$\begin{array}{ll}5.1 \text { Calculation of the sunset diagram } & 28\end{array}$

$\begin{array}{lll}5.1 .1 & \bar{\Psi}_{1} & 29\end{array}$

$\begin{array}{lll}5.1 .2 & \bar{\Psi}_{2} & 29\end{array}$

$\begin{array}{lll}5.1 .3 & \bar{\Psi}_{3} & 29\end{array}$

$\begin{array}{lll}5.1 .4 & \bar{\Psi}_{4} & 30\end{array}$

$\begin{array}{lll}5.1 .5 & \bar{\Psi}_{5} & 31\end{array}$

5.1.6 $\bar{\Psi}_{6}$ and $\bar{\Psi}_{7} \quad 32$

5.1.7 Final result for $\bar{\Psi} \quad 32$

$6 \quad$ Finite volume momentum sums with lattice regularization $\quad 32$

6.1 Some 1-loop momentum sums 33

$\begin{array}{lll}6.1 .1 & \text { Leading terms } & 34\end{array}$

6.2 Expansion coefficients 34

6.2.1 Lattice analogue of the theta function 35 
6.2.2 Asymptotic behavior for $N \rightarrow \infty \quad 37$

6.2.3 Examples: $I_{10}, I_{21}$ for $d>2 \quad 39$

6.2.4 Expansions for $d=4 \quad 42$

6.2.5 Expansions for $d=3 \quad 44$

6.2.6 Expansions for $d=2 \quad 45$

$\begin{array}{lll}6.3 & \text { Some 2-loop momentum sums } & 47\end{array}$

$\begin{array}{lll}\text { 6.3.1 } & \text { Case } d=2 & 48\end{array}$

$\begin{array}{lll}\text { 6.3.2 } & \text { Case } d=3 & 48\end{array}$

$\begin{array}{lll}\text { 6.3.3 Case } d=4 & 49\end{array}$

A Derivation of formulae for $g(0)$ and $\ddot{g}(0)$ up to $O(D-4) \quad 50$

B Evaluation of $\Psi$ for the massive case in DR 50

$\begin{array}{ll}\text { C Some useful relations involving the lattice propagator } & 53\end{array}$

$\begin{array}{ll}\text { D Modified Bessel function } & 53\end{array}$

\section{Introduction}

In an accompanying paper [1] we have computed a free energy and the mass gap in the $\delta$-regime in the framework of chiral perturbation theory without an explicit symmetry breaking term, in finite asymmetric volumes. The computation was done both in dimensional regularization (DR) and in the lattice regularization. Matching the results in two regularizations enables us to establish relations between the 4-derivative couplings appearing in these regularizations. The technical details are provided in the present paper.

In the computation of the free energy and the finite-volume mass gap we encounter one and two loop integrals over the volume. A class of 1-loop sums with dimensional regularization have been considered in detail by Hasenfratz and Leutwyler [2] in DR, we summarize some of their results and also add some more. The main part of the paper deals with the precise evaluation of the 2-loop sunset diagrams for both regularizations. For our purposes we only require diagrams with zero external momenta but the methods are more general. Massive sunset diagrams in finite volume have been treated in detail by Bijnens, Boström and Lähde [3, 4], but as far as we can tell their methods are not applicable for zero masses. In appendix B we outline an alternative method of calculating sunset diagrams by introducing a mass, in addition to the finite volume. This setup allows to take the massless limit as well.

Finally in section 6 we consider 1- and 2-loop massless sums in asymmetric finite volumes with lattice regularization. For the 1-loop sums we introduce a new method to compute precisely the coefficients of their expansions in the lattice cutoff.

The motivation to consider lattice-regularized chiral perturbation theory is addressed in [1]. 


\section{Dimensional regularization at finite volume}

Our goal is to compute massless one and two loop diagrams in finite volume with periodic boundary conditions within the framework of dimensional regularization. As in [2] we start by considering diagrams in the massive theory.

The free massive propagator is

$$
G(x, M)=\frac{1}{V} \sum_{p} \frac{\mathrm{e}^{i p x}}{p^{2}+M^{2}},
$$

where $p$ runs over a $d$-dimensional momentum space infinite lattice

$$
p=2 \pi\left(n_{0} / L_{0}, \ldots, n_{d_{s}} / L_{d_{s}}\right),
$$

where $d_{s}=d-1$ and $n_{\mu}$ are integers and

$$
V=\prod_{\mu=0}^{d_{s}} L_{\mu}
$$

The numerical evaluation of the graphs will be done for spatially cubic volumes $L_{1}=\ldots=$ $L_{d_{s}} \equiv L_{s}$, either for the hypercubic case $L_{0}=L_{s}$ or for the elongated geometry $L_{0}>L_{s}$.

We will dimensionally regularize by adding $q$ extra compact dimensions of size $\widehat{L}$ and analytically continue the resulting loop formulae to $q=-2 \epsilon$.

\subsection{Massive propagator sums in DR}

In appendices of their classic paper [2] Hasenfratz and Leutwyler considered the sums

$$
\begin{aligned}
G_{r} & =\frac{1}{V_{D}} \sum_{p} H_{r}(p), \\
H_{r}(p) & =\Gamma(r)\left(p^{2}+M^{2}\right)^{-r},
\end{aligned}
$$

where $p$ is now a $D=d+q$ dimensional vector and

$$
V_{D} \equiv V \widehat{L}^{q}
$$

For convenience, in this subsection we reproduce some of their results, in particular those that we need in [1].

Invoking the identity

$$
\frac{1}{V_{D}} \sum_{p} f(p)=\sum_{w} \tilde{f}(w)
$$

where $\widetilde{f}(x)$ is the Fourier transform of $f(p)$ :

$$
\widetilde{f}(x)=\int \frac{\mathrm{d}^{D} p}{(2 \pi)^{D}} \mathrm{e}^{i p x} f(p),
$$

and the sum over $w$ extends over the coordinate space lattice

$$
w=\left(v_{0} L_{0}, \ldots, v_{D-1} L_{D-1}\right)
$$


where $v_{k}$ are integers, one obtains

$$
G_{r}=\sum_{w} \widetilde{H}_{r}(w)
$$

with

$$
\widetilde{H}_{r}(x)=\int_{0}^{\infty} \mathrm{d} \lambda \lambda^{r-1}(4 \pi \lambda)^{-D / 2} \exp \left(-\lambda M^{2}-\frac{x^{2}}{4 \lambda}\right) .
$$

In the sum over $w$ the term $w=0$ corresponds to the infinite volume limit

$$
\widetilde{H}_{r}(0)=G_{r, \infty}=\Gamma(r) \int \frac{\mathrm{d}^{D} p}{(2 \pi)^{D}}\left(p^{2}+M^{2}\right)^{-r}=\frac{\Gamma(r-D / 2)}{(4 \pi)^{D / 2}} M^{D-2 r} .
$$

In particular

$$
G_{1, \infty}= \begin{cases}-\frac{M^{D-2}}{2 \pi}\left[\frac{1}{D-2}+\bar{C}+\frac{1}{2}\right]+\mathrm{O}(D-2) & \text { for } D-2 \sim 0 \\ -\frac{M}{4 \pi} & \text { for } D=3, \\ \frac{M^{D-2}}{8 \pi^{2}}\left[\frac{1}{D-4}+\bar{C}\right]+\mathrm{O}(D-4) & \text { for } D-4 \sim 0\end{cases}
$$

with

$$
\bar{C}=-\frac{1}{2}\left[\ln (4 \pi)-\gamma_{E}+1\right],
$$

where $\gamma_{E}=-\Gamma^{\prime}(1)=0.577 \ldots$ is the Euler constant.

It is useful to separate the infinite volume term to arrive at the representation

$$
\begin{aligned}
G_{r} & =G_{r, \infty}+g_{r}+\mathrm{O}(D-d), \\
g_{r} & =\int_{0}^{\infty} \mathrm{d} \lambda \lambda^{r-1}(4 \pi \lambda)^{-d / 2} \mathrm{e}^{-\lambda M^{2}} \sum_{v \neq 0} \exp \left(-\sum_{\mu=0}^{d_{s}} \frac{v_{\mu}^{2} L_{\mu}^{2}}{4 \lambda}\right) .
\end{aligned}
$$

In this decomposition the volume dependence is exclusively contained in the function $g_{r}$ which is unambiguous for any integer value of $D$ and is entire in $r$.

The dimensional regularization only affects the volume-independent part $G_{r, \infty}$ which contains poles at $D=2 r, 2 r+2, \ldots$

The sum occurring in the representation of $g_{r}$ converges rapidly if the sides of the box are large compared to the Compton wavelength, $M L_{\mu} \gg 1$. If all $L_{\mu} \rightarrow \infty$ then $g_{r} \rightarrow 0$ exponentially. Note if one considers other limits e.g. the temperature to $T=1 / L_{0}$ to zero i.e. $L_{0} \rightarrow \infty$ with $L_{1}, \ldots, L_{d_{s}}$ fixed, then $g_{r}$ approaches a finite limit

$$
\lim _{L_{0} \rightarrow \infty} g_{r}^{(d)}=(4 \pi)^{-1 / 2} g_{r-1 / 2}^{(d-1)} .
$$

For $r=0$ this relation reflects the fact that in the limit $T \rightarrow 0$ the partition function is dominated by the contribution from the ground state, the quantity $\ln \operatorname{det} D / 2 L_{0}$ approaching the Casimir energy associated with the $d_{s}$-dimensional box.

For the goal of considering the massless sums we need the properties of $g_{r}$ in the limit $M \rightarrow 0$ where infrared singularities occur. Introducing

$$
S(x) \equiv \sum_{n=-\infty}^{\infty} \mathrm{e}^{-\pi n^{2} x}, \quad(x>0),
$$


(related to the Jacobi theta-function, defined in (3.52)), we have

$$
g_{r}=\frac{1}{L^{d}}\left(\frac{L^{2}}{4 \pi}\right)^{r} \int_{0}^{\infty} \mathrm{d} t t^{r-d / 2-1} \exp \left(-\frac{z^{2} t}{4 \pi}\right)\left[\prod_{\mu=0}^{d_{s}} S\left(\frac{\ell_{\mu}^{2}}{t}\right)-1\right],
$$

where $L$ is some reference scale,

$$
\ell_{\mu} \equiv L_{\mu} / L
$$

and

$$
z \equiv M L
$$

We will use the shorthand notation $\underline{\ell}=\left\{\ell_{0}, \ell_{1}, \ldots, \ell_{d_{s}}\right\}$ for the relative sizes in the physical dimensions and $\hat{\ell}=\widehat{L} / L=L_{\alpha} / L$ for $\alpha=d, \ldots, D-1$ for the extra dimensions.

The dependence on the auxiliary scale $L$ of a given quantity (when the other variables are made dimensionless) is given by its dimension. Using this we will often use $L=L_{s}$ for the spatially cubic volume and denote $\ell \equiv \ell_{0}=L_{0} / L_{s}$ and $\hat{\ell}=\widehat{L} / L_{s}$.

Since the product in (2.19) often appears below we also introduce the ancillary definition (with some abuse of notation)

$$
\mathcal{S}_{d}\left(\frac{\ell^{2}}{t}\right) \equiv \prod_{\mu=0}^{d-1} S\left(\frac{\ell_{\mu}^{2}}{t}\right), \quad \mathcal{S}_{d}\left(\frac{t}{\ell^{2}}\right) \equiv \prod_{\mu=0}^{d-1} S\left(\frac{t}{\ell_{\mu}^{2}}\right) .
$$

(For the general case of $D$ dimensions we shall also use $\mathcal{S}_{D}\left(t / \ell^{2}\right)$ and $\mathcal{S}_{D}\left(\ell^{2} / t\right)$ defined analogously.)

Note that the sum in (2.18) converges very fast for $x>1$. The function $S(x)$ satisfies the well known remarkable identity

$$
S(x)=x^{-1 / 2} S\left(x^{-1}\right) .
$$

Using (2.18) for $x>1$ and (2.23) for $x<1$ one needs only a few terms in the corresponding sums to calculate $S(x)$ very precisely.

Using (2.23) one obtains the representation

$$
g_{r}=\frac{1}{L^{d}}\left(\frac{L^{2}}{4 \pi}\right)^{r}\left[A_{r}+\mathcal{V}^{-1} B_{r}-B_{r-d / 2}\right],
$$

where

$$
\begin{aligned}
& A_{r}=\frac{1}{\mathcal{V}} \int_{0}^{\infty} \mathrm{d} t t^{r-1} \exp \left(-\frac{z^{2} t}{4 \pi}\right)\left[\mathcal{S}_{d}\left(\frac{t}{\ell^{2}}\right)\right]_{\mathrm{sub}}, \\
& B_{r}=\int_{1}^{\infty} \mathrm{d} t t^{r-1} \exp \left(-\frac{z^{2} t}{4 \pi}\right),
\end{aligned}
$$

and

$$
\mathcal{V} \equiv \prod_{\mu=0}^{d_{s}} \ell_{\mu}=V L^{-d}
$$




\begin{tabular}{|l|c|c|c|c|}
\hline$d$ & 1 & 2 & 3 & 4 \\
\hline$\alpha_{-1 / 2}$ & 0.04719755120 & 0.1044122116 & 0.1750738214 & 0.2641535689 \\
$\alpha_{0}$ & 0.04619141793 & 0.1008796989 & 0.1667047726 & 0.2474072414 \\
$\alpha_{1 / 2}$ & 0.04619141793 & 0.0997350800 & 0.1627025205 & 0.2379659789 \\
$\alpha_{1}$ & 0.04719755120 & 0.1008796989 & 0.1627025205 & 0.2349151988 \\
$\alpha_{3 / 2}$ & 0.04929326270 & 0.1044122116 & 0.1667047726 & 0.2379659789 \\
$\alpha_{2}$ & 0.05265787558 & 0.1106437295 & 0.1750738214 & 0.2474072414 \\
\hline
\end{tabular}

Table 1. Values for $\alpha_{s}$ for $\ell_{\mu}=1 \forall \mu$.

In $(2.25)$ we introduced the notation $[\ldots]_{\text {sub }}$ which will be used often below. It is defined by

$$
[\Phi(t ; \ldots)]_{\mathrm{sub}}=\Phi(t ; \ldots)- \begin{cases}{[\Phi(t ; \ldots)]_{0},} & \text { for } 0<t<1 \\ {[\Phi(t ; \ldots)]_{\infty},} & \text { for } 1<t<\infty\end{cases}
$$

where $[\Phi(t ; \ldots)]_{0}$ and $[\Phi(t ; \ldots)]_{\infty}$ denote the leading asymptotic parts of $\Phi(t ; \ldots)$ at $t \rightarrow 0$ and $t \rightarrow \infty$, respectively. According to (2.18) and (2.23) one has $[S(x)]_{0}=x^{-1 / 2}$ and $[S(x)]_{\infty}=1$. In particular, in $(2.25)$ one has

$$
\left[\mathcal{S}_{d}\left(\frac{t}{\ell^{2}}\right)\right]_{0}=\mathcal{V} t^{-d / 2}, \quad\left[\mathcal{S}_{d}\left(\frac{t}{\ell^{2}}\right)\right]_{\infty}=1 .
$$

The quantity $A_{r}$ does not contain infrared singularities and has an expansion in $M^{2}$ of the form

$$
A_{r}=\sum_{n=0}^{\infty}\left(-\frac{z^{2}}{4 \pi}\right)^{n} \frac{1}{n !} \alpha_{r+n} .
$$

The expansion coefficients ${ }^{1}$ depend on $d$ and the ratios $\ell_{\mu}$ :

$$
\alpha_{s}=\frac{1}{\mathcal{V}} \int_{0}^{\infty} \mathrm{d} t t^{s-1}\left[\mathcal{S}_{d}\left(\frac{t}{\ell^{2}}\right)\right]_{\mathrm{sub}} .
$$

Some values for $\alpha_{s}$ for $\ell_{\mu}=1, \forall \mu$ are given in table 1 .

Infrared divergences are contained in the incomplete $\Gamma$-function $B_{s}$, in the form of fractional powers of $M^{2}$ :

$$
B_{s}=\left(\frac{z^{2}}{4 \pi}\right)^{-s} \Gamma(s)-\sum_{n=0}^{\infty} \frac{1}{n !}\left(-\frac{z^{2}}{4 \pi}\right)^{n} \frac{1}{n+s} .
$$

The pole in the $\Gamma$-function at $r=-N, N \in \mathbb{N}$ is canceled by a pole occurring in the piece which is analytic in $M$. Merging the two singularities one obtains a logarithmic contribution

$$
\begin{aligned}
B_{-N}= & \frac{(-1)^{N+1}}{N !}\left(\frac{z^{2}}{4 \pi}\right)^{N}\left\{\ln \left(\frac{z^{2}}{4 \pi}\right)+\gamma_{E}-\sum_{n=1}^{N} \frac{1}{n}\right\} \\
& +\sum_{n=0, n \neq N}^{\infty} \frac{1}{n !}\left(-\frac{z^{2}}{4 \pi}\right)^{n} \frac{1}{N-n}, \quad N=0,1,2, \ldots
\end{aligned}
$$

\footnotetext{
${ }^{1}$ This expression is equivalent to the one given in [2] but has a more compact form.
} 
For $z=0$ and $s<0$ one has

$$
\left.B_{s}\right|_{z=0}=-\frac{1}{s}, \quad \text { for } s<0 .
$$

The small $M$ expansions of $g_{r}$ for integer $r$ can be obtained from that of $g_{0}$ using the recursion relations

$$
g_{r+1}=-\mathrm{d} g_{r} / \mathrm{d} M^{2} .
$$

For $d=2$ the expansion of $g_{0}$ takes the form

$$
\begin{aligned}
& g_{0}=\frac{1}{L^{2}}\left[-2 \mathcal{V}^{-1} \ln (z)-\frac{z^{2}}{2 \pi} \ln (z)+\sum_{n=0}^{\infty} \frac{1}{n !} \beta_{n} z^{2 n}\right], \\
& \beta_{0}=\alpha_{0}-1+\mathcal{V}^{-1}\left\{\ln (4 \pi)-\gamma_{E}\right\}, \\
& \beta_{1}=\frac{1}{4 \pi}\left[-\alpha_{1}-\left\{-\ln (4 \pi)+\gamma_{E}-1\right\}+\mathcal{V}^{-1}\right], \\
& \beta_{n}=\left(-\frac{1}{4 \pi}\right)^{n}\left[\alpha_{n}+\frac{1}{n-1}-\frac{1}{n \mathcal{V}}\right], n \geq 2, d=2 .
\end{aligned}
$$

For $d=3$ :

$$
\begin{aligned}
& g_{0}=\frac{1}{L^{3}}\left[-2 \mathcal{V}^{-1} \ln (z)-\frac{z^{3}}{6 \pi}+\sum_{n=0}^{\infty} \frac{1}{n !} \beta_{n} z^{2 n}\right], \\
& \beta_{0}=\alpha_{0}-\frac{2}{3}+\mathcal{V}^{-1}\left\{\ln (4 \pi)-\gamma_{E}\right\} \\
& \beta_{n}=\left(-\frac{1}{4 \pi}\right)^{n}\left[\alpha_{n}+\frac{2}{2 n-3}-\frac{1}{n \mathcal{V}}\right], \quad n \neq 0, \quad d=3 .
\end{aligned}
$$

For $d=4$ :

$$
\begin{aligned}
& g_{0}=\frac{1}{L^{4}}\left[-2 \mathcal{V}^{-1} \ln (z)+\frac{z^{4}}{16 \pi^{2}}\left\{\ln (z)-\frac{1}{4}\right\}+\sum_{n=0}^{\infty} \frac{1}{n !} \beta_{n} z^{2 n}\right], \\
& \beta_{0}=\alpha_{0}-\frac{1}{2}+\mathcal{V}^{-1}\left\{\ln (4 \pi)-\gamma_{E}\right\}, \\
& \beta_{2}=\frac{1}{16 \pi^{2}}\left[\alpha_{2}-\ln (4 \pi)+\gamma_{E}-1-\frac{1}{2 \mathcal{V}}\right], \\
& \beta_{n}=\left(-\frac{1}{4 \pi}\right)^{n}\left[\alpha_{n}+\frac{1}{n-2}-\frac{1}{n \mathcal{V}}\right], \quad n \neq 0,2, \quad d=4 .
\end{aligned}
$$

The table 2 gives results for the symmetric box $\ell_{\mu}=1 \forall \mu$ and table 3 for $\ell=2$ for $d=3,4$.

\subsubsection{Sums with extra factor in the numerator}

Next we apply the methods of Hasenfratz and Leutwyler to the sums

$$
G_{r, 1}=\frac{1}{V_{D}} \sum_{p} p_{\nu}^{2} H_{r}(p), \text { no sum over } \nu \text {. }
$$




\begin{tabular}{|c|c|r|}
\hline$d$ & 3 & \multicolumn{1}{|c|}{4} \\
\hline$\beta_{0}$ & 1.45384668796818338855 & 1.70121582349712182477 \\
$\beta_{1}$ & 0.22578495944075803348 & 0.14046098554536575282 \\
$\beta_{2}$ & 0.01060752889198424526 & -0.02030477369161629693 \\
\hline
\end{tabular}

Table 2. Values for $\beta_{r}$ for $\ell_{\mu}=1 \forall \mu$ for $d=3,4$.

\begin{tabular}{|l|c|r|}
\hline$d$ & 3 & \multicolumn{1}{|c|}{4} \\
\hline$\beta_{0}$ & 0.74461239033155890201 & 0.98194779750230477518 \\
$\beta_{1}$ & 0.14370432528775141208 & 0.05911493648278131899 \\
$\beta_{2}$ & 0.02021612362190113525 & -0.01075957063969698115 \\
\hline
\end{tabular}

Table 3. Values for $\beta_{r}$ for $\ell_{\mu}=1, \mu>0$ and $\ell=2$.

We have

$$
G_{r, 1}=G_{r, 1, \infty}+g_{r, 1}+\mathrm{O}(D-d)
$$

where for infinite volume:

$$
G_{r, 1, \infty}=\Gamma(r) \int \frac{\mathrm{d}^{D} p}{(2 \pi)^{D}} p_{\nu}^{2}\left(p^{2}+M^{2}\right)^{-r}=\frac{\Gamma(r-1-D / 2)}{2(4 \pi)^{D / 2}} M^{D-2 r+2}=\frac{1}{2} G_{r-1, \infty},
$$

and

$$
\begin{aligned}
g_{r, 1} & =\frac{1}{2} g_{r-1}+\ell_{\nu}^{2} h_{r}, \\
h_{r} & =-\frac{1}{4} L^{2} \int_{0}^{\infty} \mathrm{d} \lambda \lambda^{r-3}(4 \pi \lambda)^{-d / 2} \mathrm{e}^{-\lambda M^{2}} \sum_{v} v_{\nu}^{2} \exp \left(-\sum_{\mu=0}^{d_{s}} \frac{v_{\mu}^{2} L_{\mu}^{2}}{4 \lambda}\right) .
\end{aligned}
$$

Denote the logarithmic derivative of $S(x)$ by

$$
T(x)=\frac{S^{\prime}(x)}{S(x)}=-\frac{\pi}{S(x)} \sum_{n=-\infty}^{\infty} n^{2} \mathrm{e}^{-\pi n^{2} x}
$$

satisfying the relation

$$
T(x)=-\frac{1}{2 x}-\frac{1}{x^{2}} T\left(\frac{1}{x}\right) .
$$

With this one has

$$
h_{r}=\frac{1}{L^{d}}\left(\frac{L^{2}}{4 \pi}\right)^{r-1} \int_{0}^{\infty} \mathrm{d} t t^{r-d / 2-3} \exp \left(-\frac{z^{2} t}{4 \pi}\right) T\left(\frac{\ell_{\nu}^{2}}{t}\right) \mathcal{S}_{d}\left(\frac{\ell^{2}}{t}\right) .
$$

Putting the parts together we have

$$
g_{r, 1}=\frac{1}{2} \frac{1}{L^{d}}\left(\frac{L^{2}}{4 \pi}\right)^{r-1}\left[-B_{r-1-d / 2}+D_{r}\right],
$$


where $D_{r}$ has the expansion in $z$ :

$$
D_{r}=\sum_{n=0}^{\infty}\left(-\frac{z^{2}}{4 \pi}\right)^{n} \frac{1}{n !} \gamma_{r+n},
$$

with

$$
\gamma_{s}=-\frac{2}{\ell_{\nu}^{2} \mathcal{V}} \int_{0}^{\infty} \mathrm{d} t t^{s-1}\left[T\left(\frac{t}{\ell_{\nu}^{2}}\right) \mathcal{S}_{d}\left(\frac{t}{\ell^{2}}\right)\right]_{\mathrm{sub}} .
$$

For the definition of $[\ldots]_{\text {sub }}$ see $(2.28)$. Note that $[T(x)]_{0}=-1 /(2 x)$ and $[T(x)]_{\infty}=0$.

For the totally symmetric box $\ell_{\mu}=1, \forall \mu$ we have

$$
\gamma_{s}=\frac{2}{d}(s-1) \alpha_{s-1}, \quad \ell=1 .
$$

We then have for $D \sim 3$ :

$$
G_{2,1}=\frac{1}{8 \pi L}\left[\gamma_{2}-2\right]+\mathrm{O}(D-3)+\mathrm{O}(M)
$$

and for $D \sim 4$ :

$$
\begin{aligned}
G_{2,1}= & \frac{1}{8 \pi L^{2}}\left[\gamma_{2}-1\right] \\
& +\frac{M^{2}}{16 \pi^{2}}\left[-\ln L+\frac{1}{D-4}-\frac{1}{2} \gamma_{3}\right]+\mathrm{O}(D-4)+\mathrm{O}\left(M^{4}\right),
\end{aligned}
$$

(note $\gamma_{2}$ depends on $d$ ).

\subsubsection{Keeping first order $D-d$ terms in $G_{r, 1}$}

In our computation [1] we need to keep in consideration order $q=D-d$ terms in $G_{r, 1}$ :

$$
G_{r, 1}=G_{r, 1, \infty}+\mathcal{G}_{r, 1}
$$

where

$$
\mathcal{G}_{r, 1}=\frac{1}{L^{D}}\left(\frac{L^{2}}{4 \pi}\right)^{r-1}\left[\frac{1}{2} \mathcal{G}_{r}+\ell_{\nu}^{2} \overline{\mathcal{H}}_{r}\right]
$$

with

$$
\begin{gathered}
\mathcal{G}_{r}=\int_{0}^{\infty} \mathrm{d} t t^{r-D / 2-2} \exp \left(-\frac{z^{2} t}{4 \pi}\right)\left[S\left(\frac{\hat{\ell}^{2}}{t}\right)^{q} \mathcal{S}_{d}\left(\frac{\ell^{2}}{t}\right)-1\right], \\
\overline{\mathcal{H}}_{r}=\int_{0}^{\infty} \mathrm{d} t t^{r-D / 2-3} \exp \left(-\frac{z^{2} t}{4 \pi}\right) S\left(\frac{\hat{\ell}^{2}}{t}\right)^{q} T\left(\frac{\ell_{\nu}^{2}}{t}\right) \mathcal{S}_{d}\left(\frac{\ell^{2}}{t}\right) .
\end{gathered}
$$

So

$$
\mathcal{G}_{r, 1}=(1-q \ln L) g_{r, 1}(\underline{\ell})+q \frac{1}{L^{d}}\left(\frac{L^{2}}{4 \pi}\right)^{r-1} g_{r, 1}^{(1)}(\underline{\ell}, \hat{\ell})+\mathrm{O}\left(q^{2}\right)
$$

with

$$
\begin{aligned}
g_{r, 1}^{(1)}= & -\frac{1}{\ell_{\nu}^{2} \mathcal{V}} \int_{0}^{\infty} \mathrm{d} t t^{r-1} \exp \left(-\frac{z^{2} t}{4 \pi}\right)\left[\ln \left(\frac{1}{\hat{\ell}} S\left(\frac{t}{\hat{\ell}^{2}}\right)\right) T\left(\frac{t}{\ell_{\nu}^{2}}\right) \mathcal{S}_{d}\left(\frac{t}{\ell^{2}}\right)\right]_{\mathrm{sub}} \\
& +\frac{1}{4} \int_{1}^{\infty} \mathrm{d} t t^{r-d / 2-2} \ln (t) \exp \left(-\frac{z^{2} t}{4 \pi}\right) .
\end{aligned}
$$

For the expression appearing here one has $[\ldots]_{0}=\frac{1}{4} \ell_{\nu}^{2} \mathcal{V} t^{-d / 2-1} \ln (t)$ and $[\ldots]_{\infty}=0$. 
We introduce

$$
\begin{aligned}
& \rho_{r}(\underline{\ell}, \hat{\ell})=\frac{1}{\mathcal{V}} \int_{0}^{\infty} \mathrm{d} t t^{r-1}\left[\ln \left(\frac{1}{\hat{\ell}} S\left(\frac{t}{\hat{\ell}^{2}}\right)\right) \mathcal{S}_{d}\left(\frac{t}{\ell^{2}}\right)\right]_{\mathrm{sub}}, \\
& \tau_{r}(\underline{\ell}, \hat{\ell})=-\frac{1}{\ell_{\nu}^{2} \mathcal{V}} \int_{0}^{\infty} \mathrm{d} t t^{r-1}\left[\ln \left(\frac{1}{\hat{\ell}} S\left(\frac{t}{\hat{\ell}^{2}}\right)\right) T\left(\frac{t}{\ell_{\nu}^{2}}\right) \mathcal{S}_{d}\left(\frac{t}{\ell^{2}}\right)\right]_{\mathrm{sub}} .
\end{aligned}
$$

They have a finite limit for $\hat{\ell} \rightarrow \infty$

$$
\begin{aligned}
& \rho_{r}(\underline{\ell}, \infty)=-\frac{1}{2 \mathcal{V}} \int_{0}^{\infty} \mathrm{d} t t^{r-1} \ln (t)\left[\mathcal{S}_{d}\left(\frac{t}{\ell^{2}}\right)\right]_{\mathrm{sub}}, \\
& \tau_{r}(\underline{\ell}, \infty)=\frac{1}{2 \ell_{\nu}^{2} \mathcal{V}} \int_{0}^{\infty} \mathrm{d} t t^{r-1} \ln (t)\left[T\left(\frac{t}{\ell_{\nu}^{2}}\right) \mathcal{S}_{d}\left(\frac{t}{\ell^{2}}\right)\right]_{\mathrm{sub}} .
\end{aligned}
$$

Using these for $z=0$ and $r<d / 2+1$ one obtains

$$
\left.g_{r, 1}^{(1)}\right|_{z=0}=\tau_{r}(\underline{\ell}, \hat{\ell})+\frac{1}{(d+2-2 r)^{2}} .
$$

In particular, for the case $r=1$ (cf. (2.55), (2.56))

$$
\left.g_{1,1}\right|_{z=0}=\frac{1}{2 L^{d}}\left[\gamma_{1}-\frac{2}{d}\right]
$$

and for $r=1, d=4:^{2}$

$$
\left.g_{1,1}^{(1)}\right|_{z=0, d=4} \equiv \mathcal{W}_{1}(\underline{\ell}, \hat{\ell})=\left.\tau_{1}(\underline{\ell}, \hat{\ell})\right|_{d=4}+\frac{1}{16}
$$

For $\hat{\ell} \rightarrow \infty$ one obtains

$$
\mathcal{W}_{1}(\underline{\ell}, \infty)=\left.\tau_{1}(\underline{\ell}, \infty)\right|_{d=4}+\frac{1}{16}
$$

\section{Massless propagator sums in dimensional regularization}

We are now in a position to give results for dimensionally regularized momentum sums

$$
\bar{I}_{n m} \equiv \frac{1}{V_{D}} \sum_{p}^{\prime} \frac{p_{0}^{2 m}}{\left(p^{2}\right)^{n}},
$$

which can be re-expressed in terms of the massless propagator

$$
G(x)=\frac{1}{V_{D}} \sum_{p}^{\prime} \frac{\mathrm{e}^{i p x}}{p^{2}},
$$

where the sum is over momenta $p=2 \pi\left(n_{0} / L_{0}, \ldots, n_{D-1} / L_{D-1}\right), n_{k} \in \mathbb{Z}$, and the prime on the sum means that the zero momentum is omitted: $\sum_{p}^{\prime}=\sum_{p \neq 0}$.

\footnotetext{
${ }^{2}$ The notation $\mathcal{W}_{1}$ was used in [1].
} 
We have e.g. ${ }^{3}$

$$
\begin{aligned}
& \bar{I}_{00}=-\frac{1}{V_{D}}, \\
& \bar{I}_{10}=G(0), \\
& \bar{I}_{20}=\int_{x}[G(x)]^{2}, \\
& \bar{I}_{21}=-\int_{x} G(x) \ddot{G}(x),
\end{aligned}
$$

where we have introduced the notation $\ddot{G}(x)=\partial^{2} G(x) / \partial x_{0}^{2}$.

The $\bar{I}_{n m}$ can be obtained by taking the limit of zero mass of the massive sums considered in the previous subsections. Here we will only consider the cases $m=0,1$ and for these we have ${ }^{4}$

$$
\begin{aligned}
& \bar{I}_{n 0}=\frac{1}{\Gamma(n)} \lim _{M \rightarrow 0}\left[G_{n}-\frac{\Gamma(n)}{M^{2 n} V_{D}}\right], \\
& \bar{I}_{n 1}=\frac{1}{\Gamma(n)} \lim _{M \rightarrow 0} G_{n, 1} .
\end{aligned}
$$

Now for $d=2$ :

$$
\begin{aligned}
G_{1} & =-\frac{1}{2 \pi} \ln (M)-\frac{1}{2 \pi}\left[\frac{1}{D-2}+\bar{C}+\frac{1}{2}\right]+g_{1}+\mathrm{O}(D-2), \\
G_{2} & =\frac{1}{4 \pi M^{2}}+g_{2}, \quad d=2 .
\end{aligned}
$$

For $d=3$ :

$$
\begin{aligned}
& G_{1}=-\frac{M}{4 \pi}+g_{1}, \\
& G_{2}=\frac{1}{8 \pi M}+g_{2}, \quad d=3,
\end{aligned}
$$

and for $d=4$ :

$$
\begin{aligned}
& G_{1}=\frac{M^{2}}{8 \pi^{2}}\left(\ln M+\frac{1}{D-4}+\bar{C}\right)+g_{1}+\mathrm{O}(D-4), \\
& \left.G_{2}=-\frac{1}{8 \pi^{2}}\left(\ln M+\frac{1}{D-4}+\bar{C}+\frac{1}{2}\right)+g_{2}+\mathrm{O}(D-4)\right) .
\end{aligned}
$$

The behavior of $g_{1}, g_{2}$ for $M \rightarrow 0$ is readily extracted from (2.35) and the representations (2.36), (2.40) and (2.43).

\footnotetext{
${ }^{3}$ One has $-\square G(x)=\delta^{(D)}(x)-1 / V_{D}$ hence $\bar{I}_{00}=-\square G(0)=-1 / V_{D}$ where we used that $\delta^{(D)}(0)=0$ in DR.

${ }^{4}$ The subtraction in (3.7) is the removal of singularity due to the zero mode for $M \rightarrow 0$. In (3.8) the zero mode is not singular in the massless limit.
} 
So for the corresponding massless sums we obtain for $d=2$ :

$$
\begin{aligned}
\bar{I}_{10} & =-\beta_{1}-\frac{1}{2 \pi}\left[\frac{1}{D-2}+\bar{C}-\ln L+\mathrm{O}(D-2)\right] \\
& =-\frac{1}{2 \pi}\left[\frac{1}{D-2}-\ln L-\frac{1}{2} \alpha_{1}+\frac{1}{2 \mathcal{V}}+\mathrm{O}(D-2)\right], \\
\bar{I}_{20} & =L^{2} \beta_{2} \\
\bar{I}_{21} & =-\frac{1}{4 \pi}\left[\frac{1}{D-2}-\ln L-\frac{1}{2} \gamma_{2}+\mathrm{O}(D-2)\right], \\
\bar{I}_{31} & =\frac{L^{2}}{64 \pi^{2}}\left[\gamma_{3}+1\right] .
\end{aligned}
$$

For $D=3$ :

$$
\begin{aligned}
\bar{I}_{10} & =-\beta_{1} L^{-1} \\
\bar{I}_{20} & =\beta_{2} L \\
\bar{I}_{21} & =\frac{1}{8 \pi L}\left(\gamma_{2}-2\right), \\
& =-\frac{1}{3 L} \beta_{1} \text { for } \ell_{\mu}=1 \forall \mu, \\
\bar{I}_{31} & =\frac{L}{64 \pi^{2}}\left[\gamma_{3}+2\right] .
\end{aligned}
$$

Finally for four dimensions:

$$
\begin{aligned}
\bar{I}_{10} & =-\beta_{1} L^{-2}, \\
\bar{I}_{20} & =\beta_{2}+\frac{1}{8 \pi^{2}}\left[\ln L-\frac{1}{D-4}-\bar{C}\right], \quad D \sim 4, \\
& =\frac{1}{8 \pi^{2}}\left[\ln L-\frac{1}{D-4}\right]+\bar{g}_{2}+\mathrm{O}(D-4), \\
\bar{I}_{21} & =\frac{1}{8 \pi L^{2}}\left(\gamma_{2}-1\right), \\
\bar{I}_{31} & =\frac{1}{32 \pi^{2}}\left[\ln L-\frac{1}{D-4}+\frac{1}{2} \gamma_{3}\right]+\mathrm{O}(D-4),
\end{aligned}
$$

where

$$
\bar{g}_{2}=\beta_{2}-\frac{\bar{C}}{8 \pi^{2}}=\frac{1}{16 \pi^{2}}\left[\alpha_{2}-\frac{1}{2 \mathcal{V}}\right] .
$$

For $\ell_{1}=\ell_{2}=\ell_{3}=1$ and $\ell_{0}=\ell$ we obtain

$$
\begin{array}{ll}
\bar{g}_{2}=-0.0015995623298662568192, & \text { for } \ell=1, \\
\bar{g}_{2}=0.0079456407220530589562, & \text { for } \ell=2 .
\end{array}
$$

For the totally symmetric volume we have for $d=4$ :

$$
\begin{array}{ll}
\bar{I}_{21}=\frac{1}{4} \bar{I}_{10}=-\frac{1}{4 L^{2}} \beta_{1}, & \text { for } \ell=1 \\
\bar{I}_{31}=\frac{1}{D} \bar{I}_{20}=\frac{1}{32 \pi^{2}}\left[\ln L-\frac{1}{D-4}+\frac{1}{4}\right]+\frac{1}{4} \bar{g}_{2}+\mathrm{O}(D-4), & \text { for } \ell=1 .
\end{array}
$$




\begin{tabular}{|l|r|r|r|}
\hline$\ell$ & $\gamma_{1}$ & \multicolumn{1}{|c|}{$\gamma_{2}$} & \multicolumn{1}{c|}{$\gamma_{3}$} \\
\hline 3.0 & 1.5084066631353647 & 4.4458864316201319 & 23.1870844937351901 \\
2.0 & 1.1748067357894556 & 2.3510031738406602 & 6.5174542845883118 \\
1.0 & 0 & 0.1174575993893936 & 0.2474072414293639 \\
0.5 & -10.0254579887014333 & -3.1877267490551846 & -1.3395264586420596 \\
0.25 & -167.9412483865704613 & -15.7551608044733235 & -2.7263973004934400 \\
\hline
\end{tabular}

Table 4. Values for $\gamma_{1}, \gamma_{2}, \gamma_{3}$ for $d=4, \ell_{1}=\ell_{2}=\ell_{3}=1$ and $\ell_{0}=\ell$, for different values of $\ell$.

So for the symmetric case we have

$$
\begin{array}{ll}
\gamma_{2}=1-2 \pi \beta_{1}, & \text { for } \ell=1, \\
\gamma_{3}=16 \pi^{2} \bar{g}_{2}+\frac{1}{2}, & \text { for } \ell=1 .
\end{array}
$$

and this has been checked numerically. Some values of $\gamma_{s}$ are given in table $4 .^{5}$

\subsection{The free propagator with periodic bc in DR}

Again we consider a $D$ dimensional volume with $L_{0} \equiv L_{t}, L_{1}=\cdots=L_{d-1} \equiv L_{s}$, and $L_{d}=\ldots=L_{D-1} \equiv \widehat{L}$ with periodic boundary conditions in each direction.

The goal of this section is to give the analytic basis for writing efficient programs for $G(x)$ for the numerical evaluation of classes of Feynman diagrams in coordinate space. For this purpose we find it convenient to write the finite volume propagator for the massless case, with the zero mode subtracted as

$$
G(x)=\Delta(x)+g(x),
$$

where $\Delta(x)$ is the infinite volume propagator and the finite volume piece $g(x)$ will be considered in detail in subsection 3.1.2. Note that $G(x)$ satisfies the periodic boundary conditions, while $\Delta(x)$ and $g(x)$ do not. The singularity of $G(x)$ is given entirely by $\Delta(x)$, accordingly $g(x)$ is a smooth function.

\subsubsection{Propagator in infinite volume, $D$-dimensions}

For the infinite volume propagator one has

$$
\Delta(x)=\int_{0}^{\infty} \frac{\mathrm{d} \lambda}{(4 \pi \lambda)^{D / 2}} \mathrm{e}^{-x^{2} /(4 \lambda)}=A_{D} r^{2-D},
$$

where $r=|x|$ and

$$
A_{D}=\frac{\Gamma(D / 2-1)}{4 \pi^{D / 2}}
$$

This is related to the area of the unit sphere

$$
\begin{aligned}
\Omega_{D} & =\frac{2 \pi^{D / 2}}{\Gamma(D / 2)}, \\
(D-2) A_{D} \Omega_{D} & =1 .
\end{aligned}
$$

\footnotetext{
${ }^{5}$ Due to the subtraction term $1 / t^{3}$ on the interval $t \in[0,1]$ in $(2.57)$ one has $\gamma_{1} \sim-1 /\left(2 \ell^{4}\right)$ when $\ell \ll 1$.
} 
The first and second "time" derivatives over $t=x_{0}$ are given by

$$
\begin{aligned}
& \dot{\Delta}(x)=-(D-2) A_{D} \frac{t}{r^{D}}, \\
& \ddot{\Delta}(x)=(D-2) A_{D} \frac{D t^{2}-r^{2}}{r^{D+2}} .
\end{aligned}
$$

In particular for $D=4$ one has $\Omega_{4}=2 \pi^{2}$ and

$$
\begin{aligned}
& \Delta(x)=\frac{1}{4 \pi^{2} r^{2}}, \\
& \dot{\Delta}(x)=-\frac{t}{2 \pi^{2} r^{4}}, \\
& \ddot{\Delta}(x)=\frac{4 t^{2}-r^{2}}{2 \pi^{2} r^{6}} .
\end{aligned}
$$

Note that by definition (by analytic continuation from $D<2$ ) in DR one has $\Delta(0)=0$.

\subsubsection{Calculating $g(x)$ for general $D$}

We start with the representation

$$
G(x)=\frac{1}{V_{D}} \sum_{p}^{\prime} \mathrm{e}^{i p x} \int_{0}^{\infty} \mathrm{d} \lambda \mathrm{e}^{-\lambda p^{2}} .
$$

Then following [2] we split the region according to

$$
G(x)=\int_{0}^{\mu} \frac{\mathrm{d} \lambda}{(4 \pi \lambda)^{D / 2}} \sum_{v} \mathrm{e}^{-(x+w)^{2} /(4 \lambda)}-\frac{\mu}{V_{D}}+\frac{1}{V_{D}} \sum_{p}^{\prime} \frac{\mathrm{e}^{i p x-\mu p^{2}}}{p^{2}},
$$

where we have used the Poisson summation formula in the form

$$
\frac{1}{V_{D}} \sum_{p} \mathrm{e}^{i p x-\lambda p^{2}}=\frac{1}{(4 \pi \lambda)^{D / 2}} \sum_{v} \mathrm{e}^{-(x+w)^{2} /(4 \lambda)},
$$

where

$$
w=\left(v_{0} L_{0}, \ldots, v_{D-1} L_{D-1}\right), \quad v_{\mu} \in \mathbb{Z} .
$$

For $D=4$ this gives

$$
\begin{aligned}
g(x)= & -\frac{1}{4 \pi^{2} x^{2}}\left(1-\mathrm{e}^{-x^{2} /(4 \mu)}\right)+\sum_{v}^{\prime} \frac{1}{4 \pi^{2}(x+w)^{2}} \mathrm{e}^{-(x+w)^{2} /(4 \mu)} \\
& -\frac{\mu}{V_{D}}+\frac{1}{V_{D}} \sum_{p}^{\prime} \frac{\mathrm{e}^{i p x-\mu p^{2}}}{p^{2}} . \quad(D=4)
\end{aligned}
$$

Taking $\mu=\alpha L^{2} /(4 \pi)$ with some length scale $L \approx L_{s} \approx L_{t}$ and $\alpha \approx 1$ only a few terms are needed in the sums. Of course, the final result does not depend on $\alpha$.

It is convenient to use the Jacobi theta function

$$
\begin{aligned}
S(u, z) & =\sum_{n=-\infty}^{\infty} \mathrm{e}^{-\pi u(n+z)^{2}} \\
& =u^{-1 / 2} \sum_{n=-\infty}^{\infty} \mathrm{e}^{-\pi n^{2} / u} \cos (2 \pi n z) .
\end{aligned}
$$


The first sum above converges quickly for $1 \leq u$ while the second for $0<u<1$. One has

$$
S(u, z)= \begin{cases}u^{-1 / 2}+\mathrm{O}\left(\mathrm{e}^{-\pi / u}\right), & \text { for } u \rightarrow 0 . \\ \mathrm{e}^{-\pi z^{2} u}+\mathrm{O}\left(\mathrm{e}^{-\pi u / 4}\right), & \text { for } u \rightarrow \infty,|z| \leq 1 / 2 .\end{cases}
$$

Further one has

$$
S(u, 0)=S(u), \quad \int_{0}^{1} \mathrm{~d} z S(u, z)=u^{-1 / 2} .
$$

With this one obtains (using the arbitrary scale $L$ ) the following representation for $g(x)$ suitable for numerical evaluation:

$$
g(x) L^{D-2}=\frac{1}{4 \pi} \int_{0}^{\infty} \mathrm{d} u\left\{u^{-D / 2} \prod_{\mu=0}^{D-1} S\left(\frac{\ell_{\mu}^{2}}{u}, \frac{x_{\mu}}{L_{\mu}}\right)-u^{-D / 2} \mathrm{e}^{-\pi x^{2} /\left(L^{2} u\right)}-\frac{1}{\mathcal{V}_{D}}\right\}
$$

and

$$
\begin{aligned}
g(0) L^{D-2} & =\frac{1}{4 \pi} \int_{0}^{\infty} \mathrm{d} u\left\{u^{-D / 2} \mathcal{S}_{D}\left(\frac{\ell^{2}}{u}\right)-u^{-D / 2}-\frac{1}{\mathcal{V}_{D}}\right\} \\
& =\frac{1}{4 \pi} \int_{0}^{\infty} \mathrm{d} u\left\{\frac{1}{\mathcal{V}_{D}} \mathcal{S}_{D}\left(\frac{u}{\ell^{2}}\right)-u^{-D / 2}-\frac{1}{\mathcal{V}_{D}}\right\}
\end{aligned}
$$

In our computations we will need the expansion of $g(0)$ and $\partial_{\nu}^{2} g(0)$ in $q=D-d$ up to first order:

$$
\begin{aligned}
& g(0) L^{D-2}=g_{0}(0)+q g_{1}(0)+\mathrm{O}\left(q^{2}\right) \\
& \partial_{\nu}^{2} g(0) L^{D}=\partial_{\nu}^{2} g_{0}(0)+q \partial_{\nu}^{2} g_{1}(0)+\mathrm{O}\left(q^{2}\right) .
\end{aligned}
$$

Using (2.31) and (2.67) one gets for $d>2$

$$
\begin{aligned}
& g_{0}(0)=\frac{1}{4 \pi}\left[\alpha_{1}(\underline{\ell})-\frac{2}{d-2}-\frac{1}{\mathcal{V}}\right], \\
& g_{1}(0)=\frac{1}{4 \pi}\left[\rho_{1}(\underline{\ell}, \hat{\ell})+\frac{2}{(d-2)^{2}}+\frac{\ln (\hat{\ell})}{\mathcal{V}}\right] .
\end{aligned}
$$

Some values of $g_{0}(0), g_{1}(0)$ for $d=4$ are given in table 5 . For $d=4, L_{t} \gg L_{s}$ one has $g(0) L_{s}^{2}=-0.2257849591+L_{t} /\left(12 L_{s}\right)$, and $g(t, \underline{x})$ decreases exponentially fast with $t$ until $g\left(t=L_{t} / 2, \underline{0}\right)=-\pi^{2}\left(L_{s} / L_{t}\right)^{2}+L_{t} /\left(12 L_{s}\right)$.

For the double derivatives one gets similarly (cf. appendix A)

$$
\begin{aligned}
& \partial_{\nu}^{2} g_{0}(0)=-\frac{1}{2} \gamma_{1}(\underline{\ell})+\frac{1}{d}, \\
& \partial_{\nu}^{2} g_{1}(0)=-\tau_{1}(\underline{\ell}, \hat{\ell})-\frac{1}{d^{2}} .
\end{aligned}
$$

Some values of $\partial_{\nu}^{2} g_{0}(0), \partial_{\nu}^{2} g_{1}(0)$ for $d=4$ obtained from (3.61), and (3.62) are given in tables 6,7 at different $\nu, \ell$ and $\hat{\ell}$. Here $\nu=0,1, x$ denotes the time, one of the spatial coordinates, and one of the $D-4$ auxiliary coordinates, respectively. The coefficients 


\begin{tabular}{|c|c|c|c|}
\hline$\ell$ & $\hat{\ell}$ & $g_{0}(0)$ & $g_{1}(0)$ \\
\hline 1 & 1 & -0.140460985545365753 & 0.04602401621995892 \\
1 & 2 & & 0.17329328071528666 \\
1 & 3 & & 0.38989530371165459 \\
1 & 4 & & 0.69314718058471526 \\
1 & 10 & & 4.33216987849965818 \\
1 & 15 & & 9.74738222662423091 \\
\hline 2 & 1 & -0.059114936482781319 & 0.04524519886669500 \\
2 & 2 & & 0.09156830194292184 \\
2 & 3 & & 0.19876546078660153 \\
2 & 4 & & 0.35033070353224446 \\
2 & 10 & & 2.16983884163206161 \\
2 & 15 & & 4.87744501569431526 \\
\hline 3 & \multicolumn{2}{|c|}{0.0242150467817175} & \\
4 & & 0.1075483739041890 & \\
10 & & 0.6075483738925662 & \\
20 & \multicolumn{2}{|c}{1.4408817072258595} & \\
\hline
\end{tabular}

Table 5. Numerical values of $g_{0}(0 ; \ell), g_{1}(0 ; \ell, \hat{\ell})$ for $d=4$ at different values of $\ell$ and $\hat{\ell}$. For large $\ell$ one has $g^{(d=4)}(0 ; \ell)=g^{(d=3)}(0 ; 1)+\ell / 12$, where $g^{(d=3)}(0 ; \ell=1)=-0.22578495944$.

satisfy the relations (3.64), (3.65). For large $\ell$ one has $\partial_{0}^{2} g_{0}(0, \ell)=\partial_{0}^{2} g_{0}(0, \infty)+1 / \ell$, up to an exponentially small correction.

Since $g(x)$ satisfies the relation $\square g(x)=1 / V_{D}$, one obtains for $d=4$,

$$
\partial_{0}^{2} g(0)+3 \partial_{1}^{2} g(0)+(D-4) \partial_{x}^{2} g(0)=\frac{1}{\ell \hat{\ell}^{D-4}} .
$$

Expanding in $D-4$ this leads to

$$
\begin{aligned}
\partial_{0}^{2} g_{0}(0)+3 \partial_{1}^{2} g_{0}(0) & =\frac{1}{\ell}, \\
\partial_{0}^{2} g_{1}(0)+3 \partial_{1}^{2} g_{1}(0)+\partial_{x}^{2} g_{0}(0) & =-\frac{\ln \hat{\ell}}{\ell} .
\end{aligned}
$$

The values given in table 6,7 satisfy these relations. It turns out (as expected) that $\partial_{1}^{2} g_{0}(0 ; \ell)$ approaches the $\ell \rightarrow \infty$ limit very fast.

In $D=4$ one has for small $x$

$$
g(x)=g(0)+\frac{1}{6}\left(\ddot{g}(0)-\frac{1}{4 \ell}\right)\left(4 t^{2}-x^{2}\right)+\frac{1}{8 \ell} x^{2}+\mathrm{O}\left(x^{4}\right) .
$$

\subsection{Evaluation of some 1-loop integrals using the representation in subsec- tion 3.1}

As an illustration of the coordinate method that we will use to compute the sunset diagrams in section 4 , we first apply them to the computation of $\int_{x} G(x)^{2}$ for $d=4$. The latter was 


\begin{tabular}{|c|c|c|c|c|}
\hline$\nu$ & $\ell$ & $\hat{\ell}$ & $\partial_{\nu}^{2} g_{0}(0 ; \ell)$ & $\partial_{\nu}^{2} g_{1}(0 ; \ell, \hat{\ell})$ \\
\hline 0 & 1 & 1 & 0.25 & -0.0625 \\
0 & 1 & 2 & & -0.03163874689457073 \\
0 & 1 & 3 & & -0.03157423948306426 \\
0 & 1 & 15 & & -0.03157409482132951 \\
\hline 0 & 2 & 1 & -0.33740336789472781 & -0.10189378657955458 \\
0 & 2 & 2 & & 0.08549134113439395 \\
0 & 2 & 3 & & 0.09641825496707322 \\
0 & 2 & 15 & & 0.09704905965741384 \\
0 & 3 & & -0.5042033315676696 & \\
0 & 4 & & -0.5875369102375624 & \\
0 & 10 & & -0.7375369106960411 & \\
0 & 20 & & -0.7875369106959981 & \\
0 & $\infty$ & $\infty$ & -0.83753691069608 & \\
\hline 1 & 1 & 1 & & \\
1 & 1 & 2 & & \\
1 & 1 & 3 & & -0.03163874689457073 \\
1 & 1 & 15 & & -0.03157423948306426 \\
\hline 1 & 2 & 1 & 0.27913445596490927 & -0.05908022312845156 \\
1 & 2 & 2 & & -0.03155385450654626 \\
1 & 2 & 3 & & -0.03150918768108555 \\
1 & 2 & 15 & & -0.03150910214106698 \\
1 & 3 & & 0.279178888300334 & \\
1 & 4 & & 0.279178970079188 & \\
1 & $\infty$ & & 0.279178970232028 & \\
\hline & & & \\
\hline
\end{tabular}

Table 6. Numerical values of $\partial_{\nu}^{2} g_{0}(0 ; \ell), \partial_{\nu}^{2} g_{1}(0 ; \ell, \hat{\ell})$ for $\nu=0,1$.

already treated in [2] using the momentum-space representation and the result presented in (3.26). We evaluate this Feynman graph in position-space using the decomposition $G(x)=\Delta(x)+g(x)$ and writing the result as a sum of different terms, each of which is calculated using appropriate methods, e.g. using subtraction, splitting the integration domain, etc. Comparing the result with the momentum space result of [2] is useful as a test of some subroutines that were used for numerical evaluation of the sunset diagram treated in section 4 .

\subsection{1 $\int_{x}[G(x)]^{2}$}

We start (taking $L_{s}=1$ ) by separating the cube $V_{0}=[-1 / 2,1 / 2]^{4} \times[-\hat{\ell} / 2, \hat{\ell} / 2]^{D-4}$ from the total volume $V=[-\ell / 2, \ell / 2] \times[-1 / 2,1 / 2]^{3} \times[-\hat{\ell} / 2, \hat{\ell} / 2]^{D-4}$. The splitting is illustrated in figure 1. Further we decompose

$$
\int_{V}[G(x)]^{2}=\sum_{r=1}^{5} \Sigma_{r},
$$




\begin{tabular}{|c|c|c|c|c|}
\hline$\nu$ & $\ell$ & $\hat{\ell}$ & $\partial_{\nu}^{2} g_{0}(0 ; \ell)$ & $\partial_{\nu}^{2} g_{1}(0 ; \ell, \hat{\ell})$ \\
\hline $\mathrm{x}$ & 1 & 1 & 0.25 & -0.0625 \\
$\mathrm{x}$ & 1 & 2 & -0.56659219298166237 & 0.17118801988367523 \\
$\mathrm{x}$ & 1 & 3 & -0.97231532913191441 & 0.53453238828804972 \\
$\mathrm{x}$ & 1 & 4 & -1.25998268327401261 & 0.89196426717182040 \\
$\mathrm{x}$ & 1 & 10 & -1.91028900870890396 & 2.58200729545875239 \\
$\mathrm{x}$ & 1 & 15 & -1.92086060450589695 & 3.59782618606441357 \\
\hline $\mathrm{x}$ & 2 & 1 & 0.27913445596490927 & -0.05908022312845156 \\
$\mathrm{x}$ & 2 & 2 & -0.33740336789472781 & 0.08549134113439395 \\
$\mathrm{x}$ & 2 & 3 & -0.55119683545590228 & 0.27446576838970181 \\
$\mathrm{x}$ & 2 & 4 & -0.69562959457825228 & 0.45372865145015078 \\
$\mathrm{x}$ & 2 & 10 & -1.02081444723100106 & 1.29878409706335203 \\
$\mathrm{x}$ & 2 & 15 & -1.02610024512982040 & 1.80669354236667127 \\
\hline
\end{tabular}

Table 7. Numerical values of $\partial_{\nu}^{2} g_{0}(0 ; \ell), \partial_{\nu}^{2} g_{1}(0 ; \ell, \hat{\ell})$ for the second derivative taken along one of the "extra" dimensions.

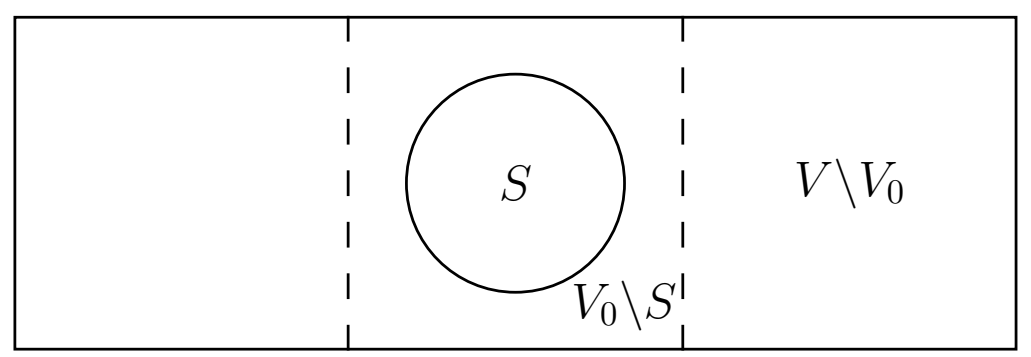

Figure 1. Splitting the regions of integration for the torus of volume $V=L_{t} \times L_{s}^{d_{s}} \times \hat{L}^{q}$. From $V$ one cuts out the hypercube $V_{0}$ and within this a $D=d+q$ dimensional sphere $S$ with the radius $\rho L_{s}$. The singularity in $D$ appears only in the integral over $S$, hence in the integrals over $V_{0} \backslash S$ and $V \backslash V_{0}$ are evaluated in $d$ dimensions. In the figure the longest direction is $L_{t}$, the other $D-1$ dimensions $L_{\mu}$ are symbolized by the vertical extent of the box.

with

$$
\begin{aligned}
& \Sigma_{1}=\int_{V_{0}}[\Delta(x)]^{2}, \quad \Sigma_{2}=2 g(0) \int_{V_{0}} \Delta(x), \quad \Sigma_{3}=2 \int_{V_{0}} \Delta(x)[g(x)-g(0)], \\
& \Sigma_{4}=\int_{V_{0}}[g(x)]^{2}, \quad \Sigma_{5}=\int_{V \backslash V_{0}}[G(x)]^{2} .
\end{aligned}
$$

To separate the divergent terms we will also need integrals over the $d$-dimensional sphere $S$ with radius $\rho$, (which will be taken to be $1 / 2$ in the actual numerical calculations) as well as integrals over $V_{0} \backslash S$.

$$
\Sigma_{1}=\Sigma_{1}^{(a)}+\Sigma_{1}^{(b)}
$$




\begin{tabular}{|c|c|c|}
\hline integral & $\ell=1$ & $\ell=2$ \\
\hline$\Sigma_{2}$ & -0.030504553273361740 & -0.012838260547502574 \\
$\Sigma_{3}$ & 0.005284317053948634 & 0.002330969017104402 \\
$\Sigma_{4}$ & 0.01189047875442587 & 0.002457080245220858 \\
$\Sigma_{5}$ & 0 & 0.004265656872109587 \\
$\Sigma$ & $-0.0015995623298662+$ & 0.0079456407220533 \\
\hline
\end{tabular}

Table 8. Values for $\Sigma_{i}$ and $\Sigma$ for $\ell=1$ and $\ell=2$.

with

$$
\begin{aligned}
\Sigma_{1}^{(a)} & =\int_{S}[\Delta(x)]^{2}=A_{D}^{2} \int_{0}^{\rho} \frac{\Omega_{D} r^{D-1} \mathrm{~d} r}{r^{2 D-4}}=-\frac{\Gamma(D / 2-1) \rho^{4-D}}{4 \pi^{D / 2}(D-2)(D-4)} \\
& =-\frac{1}{8 \pi^{2}}\left[\frac{1}{D-4}-\ln \rho-\frac{1}{2}\left(1+\gamma_{E}+\ln \pi\right)\right]+\ldots \\
& =-\frac{1}{8 \pi^{2}(D-4)}+0.00845810996666960998+\mathrm{O}(D-4),
\end{aligned}
$$

with $\rho=1 / 2$. The other contribution can be computed numerically to high precision:

$$
\Sigma_{1}^{(b)}=\int_{V_{0} \backslash S}[\Delta(x)]^{2}=0.003272085168451168, \quad\left(\rho=\frac{1}{2}, d=4\right) .
$$

So adding the two contributions we obtain

$$
\Sigma_{1}=-\frac{1}{8 \pi^{2}(D-4)}+0.011730195135121+\mathrm{O}(D-4) .
$$

Numerical integration gives

$$
\int_{V_{0}} \Delta(x)=0.1085872819235967, \quad(d=4) .
$$

The other terms $\Sigma_{3}, \Sigma_{4}, \Sigma_{5}$ can also be computed precisely for $d=4$ for arbitrary $\ell$. Finally one gets (restoring the dimensions)

$$
\int_{V}[G(x)]^{2}=-\frac{1}{8 \pi^{2}}\left\{\frac{1}{D-4}-\ln L\right\}+\Sigma(\ell)+\mathrm{O}(D-4) .
$$

Values for $\Sigma(\ell)$ and $\Sigma_{i}$ for $\ell=1, \ell=2$ are given in table 8 . We should have $\Sigma(\ell)=\bar{g}_{2}$ with $\bar{g}_{2}$ defined in (3.30). The $\ell=1$ result agrees with Hasenfratz and Leutwyler [2] (cf. their eqs. (C.5), (A.17)) also in (3.31) and for $\ell=2$ in (3.32).

\subsection{2 $\int_{x} G(x) \ddot{G}(x)$}

Next we compute $\int G \ddot{G}$ in a similar way:

$$
\int_{V} G(x) \ddot{G}(x)=\sum_{r=1}^{7} \Sigma_{r}^{\prime},
$$




\begin{tabular}{|c|l|c|}
\hline integral & \multicolumn{1}{|c|}{$\ell=1$} & $\ell=2$ \\
\hline$\Sigma_{2}^{\prime}$ & 0.027146820480899175 & -0.036637714631555824279 \\
$\Sigma_{3}^{\prime}$ & 0 & 0.004861055079412517 \\
$\Sigma_{4}^{\prime}$ & 0.035115246386341438250 & 0.014778734120695329750 \\
$\Sigma_{5}^{\prime}$ & 0 & -0.01377640717436285 \\
$\Sigma_{6}^{\prime}$ & -0.027146820480899175 & 0.00776222484407871 \\
$\Sigma_{7}^{\prime}$ & 0 & -0.03074260054253895 \\
$\Sigma^{\prime}$ & 0.0351152463863414 & -0.0537547083042711 \\
\hline
\end{tabular}

Table 9. Values for $\Sigma_{i}^{\prime}$ and $\Sigma^{\prime}$ for $\ell=1$ and $\ell=2$.

with

$$
\begin{array}{lll}
\Sigma_{1}^{\prime} & =\int_{V_{0}} \Delta(x) \ddot{\Delta}(x), \quad \Sigma_{2}^{\prime}=\ddot{g}(0) \int_{V_{0}} \Delta(x), & \Sigma_{3}^{\prime}=\int_{V_{0}} \Delta(x)[\ddot{g}(x)-\ddot{g}(0)], \\
\Sigma_{4}^{\prime}=g(0) \int_{V_{0}} \ddot{\Delta}(x), \quad \Sigma_{5}^{\prime}=\int_{V_{0}}[g(x)-g(0)] \ddot{\Delta}(x), \quad \Sigma_{6}^{\prime}=\int_{V_{0}} g(x) \ddot{g}(x) \\
\Sigma_{7}^{\prime}=\int_{V \backslash V_{0}} G(x) \ddot{G}(x) .
\end{array}
$$

Now $\Sigma_{1}^{\prime}=0$ since it is proportional to $\Delta(0)$ (which is zero in DR), and

$$
\Sigma_{4}^{\prime}=-g(0) / 4+\mathrm{O}(D-4) .
$$

The others need numerical evaluation (note the integral appearing in $\Sigma_{2}^{\prime}$ is given in (3.73)). Adding the contributions we get the desired result

$$
\int_{V} G(x) \ddot{G}(x)=\frac{1}{L^{2}} \Sigma^{\prime}(\ell)+\mathrm{O}(D-4) .
$$

Numerical values for $\ell=1,2$ are given in table 9. We should find agreement with (3.28) i.e. $\Sigma^{\prime}(\ell)=-\frac{1}{8 \pi}\left(\gamma_{2}-1\right)$. The value for $\ell=1$ agrees with $\Sigma^{\prime}(1)=-g(0)_{\ell=1} / 4{ }^{6}$ and for $\ell=2$ with the value obtained from table 4 to 10 significant digits.

\section{Massless sunset diagram with DR}

In this section we shall calculate the dimensionless quantity ${ }^{7}$

$$
\Psi=-L^{2 D-4} \bar{W}=L^{2 D-4} \int_{V} \mathrm{~d}^{D} x G(x)^{2} \ddot{G}(x),
$$

at $D \sim d=4$. Below we put $L=L_{s}=1$ for simplicity. Inserting $G(x)=\Delta(x)+g(x)$ one gets seven terms

$$
\Psi=\sum_{r=1}^{7} \Psi_{r},
$$

\footnotetext{
${ }^{6}$ Note for $\ell=1$ we have $\Sigma_{6}^{\prime}=\frac{1}{4} \int_{V_{0}} g(x)=-\frac{1}{4} \int_{V_{0}} \Delta(x)$.

${ }^{7}$ The notation $\bar{W}$ was used in eq. (3.47) of [1].
} 
with

$$
\begin{array}{llll}
\Psi_{1} & =\int_{V_{0}} g(x)^{2} \ddot{g}(x), & \Psi_{2}=\int_{V_{0}} g(x)^{2} \ddot{\Delta}(x), & \Psi_{3}=2 \int_{V_{0}} \Delta(x) g(x) \ddot{g}(x), \\
\Psi_{4}=\int_{V_{0}} \Delta(x)^{2} \ddot{g}(x), & \Psi_{5}=2 \int_{V_{0}} \Delta(x) g(x) \ddot{\Delta}(x), & \Psi_{6}=\int_{V_{0}} \Delta(x)^{2} \ddot{\Delta}(x), \\
\Psi_{7}=\int_{V \backslash V_{0}} G(x)^{2} \ddot{G}(x) . & &
\end{array}
$$

At $D=4$ only $\Psi_{4}$ and $\Psi_{5}$ have a pole, the others are finite.

\section{$4.1 \quad \Psi_{1}$}

Numerical integration of $\Psi_{1}$ is simple and results for $\ell=1, \ell=2$ are given in table 10 . Note as a check at $\ell=1$ we have $\Psi_{1}=\frac{1}{4} \Sigma_{4}=0.01189047875442588 / 4=0.002972619688606468$ which agrees to all digits.

\section{$4.2 \quad \Psi_{2}$}

We split $\Psi_{2}$ in two terms

$$
\Psi_{2}=\Psi_{2 a}+\Psi_{2 b},
$$

with

$$
\begin{aligned}
& \Psi_{2 a}=g(0)^{2} \int_{V_{0}} \ddot{\Delta}(x), \\
& \Psi_{2 b}=\int_{V_{0}}\left[g(x)^{2}-g(0)^{2}\right] \ddot{\Delta}(x) .
\end{aligned}
$$

Now

$$
\int_{V_{0}} \ddot{\Delta}(x)=\frac{1}{D} \int_{V_{0}} \square \Delta(x)=-\frac{1}{D}
$$

hence

$$
\Psi_{2 a}=-\frac{1}{4} g(0)^{2} .
$$

$\Psi_{2 b}$ is zero for $\ell=1$. A direct calculation for $\ell=2$ in Cartesian coordinates is not too precise, and we get $\Psi_{2 b}=0.001458 \ldots, \quad \ell=2$. The reason for poor convergence is the integrable singularity at the origin. One can improve drastically the convergence by changing the variables

$$
x_{0}=\eta, \quad x_{i}=\eta u_{i}, \quad i=1,2,3 .
$$

The region $\eta \in[0,1 / 2], u_{i} \in[-1,1]$ corresponds to the pyramid with the hyper-face $x_{0}=+1 / 2$ as basis. ${ }^{8}$ The change of variables is illustrated in figure 2. Precise values of $\Psi_{2 a}, \Psi_{2 b}$ for $\ell=1, \ell=2$ are given in table 10.

${ }^{8}$ These variables are also convenient to describe the part of the pyramid cut out by sphere $S$ : $1 /\left(2 \sqrt{1+\mathbf{u}^{2}}\right) \leq \eta \leq 1 / 2$. 


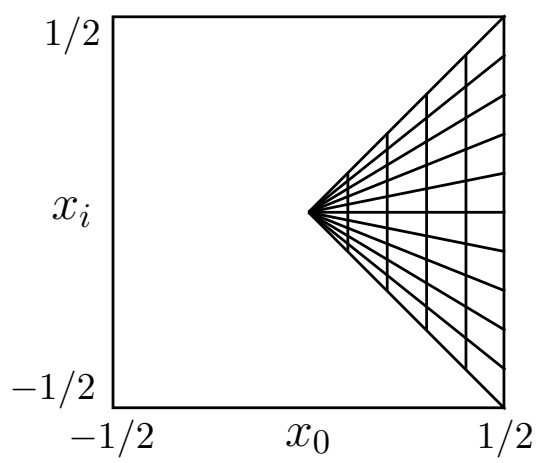

Figure 2. For integration over the hypercube $V_{0}=[-1 / 2,1 / 2]^{d}$ or over $V_{0} \backslash S$ it is convenient to split $V_{0}$ into $2 d$ pyramids and introduce in each of them new variables $\eta$ and $u_{i}$ where $x_{0}=\eta$ and $x_{i}=\eta u_{i}$ (cf. (4.9)). This trick improves the precision of integration in the case when one has an integrable singularity at $x=0$.

\section{$4.3 \quad \Psi_{3}$}

We also split $\Psi_{3}$ in two parts

$$
\Psi_{3}=\Psi_{3 a}+\Psi_{3 b}
$$

with

$$
\begin{aligned}
\Psi_{3 a} & =2 g(0) \ddot{g}(0) \int_{V_{0}} \Delta(x), \\
\Psi_{3 b} & =2 \int_{V_{0}} \Delta(x)[g(x) \ddot{g}(x)-g(0) \ddot{g}(0)] .
\end{aligned}
$$

All quantities appearing in $\Psi_{3 a}$ are already available, and there are no problems with numerical computation of $\Psi_{3 b}$ for $d=4$. Values of $\Psi_{3 a}, \Psi_{3 b}$ for $\ell=1, \ell=2$ are given in table 10. As a check, for $\ell=1$ one has $\Psi_{3 b}=\frac{1}{4} \Sigma_{3}$ which gives the same value as in table 10 .

\section{$4.4 \quad \Psi_{4}$}

We have

$$
\Psi_{4}=\Psi_{4 a}+\Psi_{4 b},
$$

with

$$
\begin{aligned}
\Psi_{4 a} & =\ddot{g}(0) \Sigma_{1}, \\
\Psi_{4 b} & =\int_{V_{0}} \Delta(x)^{2}[\ddot{g}(x)-\ddot{g}(0)] .
\end{aligned}
$$

Note that in eq. (4.14) $\Sigma_{1}$ is given in (3.72) and because of the pole one needs $\ddot{g}_{1}(0)$, the $\mathrm{O}(D-4)$ term in $\ddot{g}(0)$ which is given in eq. (3.62).

Adding values for $\Psi_{4 b}$ we have

$$
\Psi_{4}(\ell, \hat{\ell})=-\frac{\ddot{g}_{0}(0 ; \ell)}{8 \pi^{2}(D-4)}-\frac{\ddot{g}_{1}(0 ; \ell, \hat{\ell})}{8 \pi^{2}}+\Psi_{4 c} .
$$

Here $\Psi_{4 c}$ is the sum of $\Psi_{4 b}$ and the contribution from the constant term in (3.72). Values for $\Psi_{4 b}, \Psi_{4 c}$ are given in table 10 . 


\section{$4.5 \quad \Psi_{5}$}

As for $\Sigma_{1}$ to separate the divergent terms we separate from the volume a $D$-dimensional sphere $S$ with radius $\rho$ :

$$
\Psi_{5}=\Psi_{5 a}+\Psi_{5 b}+\Psi_{5 c}+\Psi_{5 d},
$$

with

$$
\begin{aligned}
\Psi_{5 a} & =2 g(0) \int_{S} \Delta(x) \ddot{\Delta}(x), \\
\Psi_{5 b} & =\sum_{\nu} \partial_{\nu}^{2} g(0) \psi_{5 b}^{(\nu)}, \\
\psi_{5 b}^{(\nu)} & =\int_{S} x_{\nu}^{2} \Delta(x) \ddot{\Delta}(x), \\
\Psi_{5 c} & =2 \int_{S} \hat{g}(x) \Delta(x) \ddot{\Delta}(x), \quad \hat{g}(x)=g(x)-g(0)-\frac{1}{2} \sum_{\nu} x_{\nu}^{2} \partial_{\nu}^{2} g(0), \\
\Psi_{5 d} & =2 \int_{V_{0} \backslash S} \ddot{\Delta}(x) \Delta(x) g(x) .
\end{aligned}
$$

$\Psi_{5 a}$ is zero since by symmetry it is proportional to $\Delta(0)$.

Next for $\nu=0$ we have

$$
\begin{aligned}
\psi_{5 b}^{(0)} & =\int_{S} \ddot{\Delta}(x) \Delta(x) t^{2}=(D-2) A_{D}^{2} \int_{S} \frac{\left(D t^{2}-r^{2}\right) t^{2}}{r^{2 D}} \\
& =(D-2) A_{D}^{2} \Omega_{D} \int_{0}^{\rho} \frac{\mathrm{d} r}{r^{D-3}}\left\langle D \cos ^{4} \vartheta-\cos ^{2} \vartheta\right\rangle,
\end{aligned}
$$

where $\rho=1 / 2$. The averaging over $\vartheta$ is with weight $\sin ^{D-2} \vartheta$. One has

$$
I_{D}=\int_{0}^{\pi} \sin ^{D-2} \vartheta \mathrm{d} \vartheta=\frac{\Gamma(1 / 2) \Gamma(D / 2-1 / 2)}{\Gamma(D / 2)}
$$

and $\Omega_{D}=\Omega_{D-1} I_{D}$. For general $n$ we have

$$
\left\langle\cos ^{n} \vartheta\right\rangle=\frac{\Gamma(n / 2+1 / 2) \Gamma(D / 2)}{\Gamma(n / 2+D / 2) \Gamma(1 / 2)} .
$$

The averages appearing above are

$$
\begin{aligned}
\left\langle\cos ^{2} \vartheta\right\rangle & =\frac{1}{D}, \\
\left\langle\cos ^{4} \vartheta\right\rangle & =\frac{3}{D(D+2)} .
\end{aligned}
$$

Since $\int_{S} \ddot{\Delta}(x) \Delta(x) r^{2}=0$ by symmetry, we obtain

$$
\psi_{5 b}^{(\nu)}=\frac{2^{D-5} \Gamma(D / 2-1)}{\pi^{D / 2}(D-4) D(D+2)}\left(1-D \delta_{\nu 0}\right) .
$$

Using eq. (3.63) one has

$$
\sum_{\nu} \partial_{\nu}^{2} g(0)\left(1-D \delta_{\nu 0}\right)=\frac{1}{\ell \hat{\ell}^{D-4}}-D \ddot{g}(0) .
$$


This gives

$$
\Psi_{5 b}=\frac{2^{D-5} \Gamma(D / 2-1)}{\pi^{D / 2} D(D+2)(D-4)}\left[\frac{1}{\ell \hat{\ell}^{D-4}}-D \ddot{g}(0)\right] .
$$

Because of the pole at $D=4$ we need here the expansion of $\ddot{g}(0)$ to first order in $D-4$, given in eq. (3.62) and table 6.

Expanding in $D-4$ one finally has

$$
\begin{aligned}
\Psi_{5 b}(\ell, \hat{\ell})= & \frac{1}{48 \pi^{2}}\left(\frac{1}{D-4}+\ln 2-\frac{1}{2} \gamma-\frac{1}{2} \ln \pi-\frac{1}{6}\right)\left(\frac{1}{\ell}-4 \ddot{g}_{0}(0 ; \ell)\right) \\
& -\frac{1}{48 \pi^{2}}\left(\frac{\ln \hat{\ell}}{\ell}+\frac{1}{4 \ell}+4 \ddot{g}_{1}(0 ; \ell, \hat{\ell})\right)+\mathrm{O}(D-4) .
\end{aligned}
$$

As a check one can verify that $\Psi_{5 b}(1,1)=0$ since $\ddot{g}_{0}(0 ; 1)=1 / 4$ and $\ddot{g}_{1}(0 ; 1,1)=-1 / 16$.

The last two integrals are convergent. Note that in $d=4$ one has

$$
g(x)=g(0)+\frac{1}{6}\left(\ddot{g}(0)-\frac{1}{4 \ell}\right) .
$$

The integral in $\Psi_{5 c}$ is convergent due to the subtraction $\left(\hat{g}(x)=\mathrm{O}\left(r^{4}\right)\right)$ and it is obviously zero for $\ell=1$ due to cubic symmetry. Numerical integration with increasing precision indicates convergence to zero also for $\ell>1$ although $\hat{g}(x)$ does not have the cubic symmetry in this case. A closer look shows that the angular integration at fixed $r$ gives zero. This can be explained as follows. Since $\square g(x)=1 / \ell$ it follows from (4.21) that $\hat{g}(x)$ is a harmonic function, $\square \hat{g}(x)=0$. Further, since $\hat{g}(x)=\mathrm{O}\left(r^{4}\right)$ its expansion in powers of $r$ contains only spherical harmonics of order larger than two. The angular dependence of $\ddot{\Delta}(x) \propto 4 t^{2}-x^{2}$ in $\Psi_{5 c}$ is given by a spherical harmonics of order two, hence due to the orthogonality of the spherical harmonics the angular integration indeed gives zero.

Hence we conclude that

$$
\Psi_{5 c}=0
$$

Adding together $\Psi_{5}=\Psi_{5 b}+\Psi_{5 d}$ one has

$\Psi_{5}(\ell, \hat{\ell})=\frac{1}{48 \pi^{2}(D-4)}\left(\frac{1}{\ell}-4 \ddot{g}_{0}(0 ; \ell)\right)-\frac{1}{48 \pi^{2}}\left(\frac{\ln \hat{\ell}}{\ell}+\frac{1}{4 \ell}+4 \ddot{g}_{1}(0 ; \ell, \hat{\ell})\right)+\psi_{5}+\mathrm{O}(D-4)$.

Values of $\Psi_{5 d}$ and $\psi_{5}$ for $\ell=1, \ell=2$ are given in table $10,\left(\Psi_{5 d}=0\right.$ for $\ell=1, d=4$ because of symmetry).

\section{6 $\quad \Psi_{6}$ and $\Psi_{7}$}

The integral $\Psi_{6}$ is zero

$$
\Psi_{6}=\int_{V_{0}} \Delta(x)^{2} \ddot{\Delta}(x)=-\frac{1}{D} \Delta(0)^{2}=0 .
$$

Also $\Psi_{7}=0$ for $\ell=1$ and its value for $\ell=2$ is given in table 10 . 


\subsection{The final result for $\Psi$}

Collecting all terms one gets ${ }^{9}$ for the sunset diagram eq. (4.1)

$$
\begin{aligned}
\Psi(\ell, \hat{\ell}) & =-\frac{1}{48 \pi^{2}(D-4)}\left(10 \ddot{g}(0 ; \ell)-\frac{1}{\mathcal{V}_{D}}\right)-\frac{1}{16 \pi^{2}} \overline{\mathcal{W}}(\ell)+\mathrm{O}(D-4) \\
& =-\frac{1}{48 \pi^{2}}\left[\frac{1}{(D-4)}\left\{10 \ddot{g}_{0}(0 ; \ell)-\frac{1}{\ell}\right\}+10 \ddot{g}_{1}(0 ; \ell, \hat{\ell})+\frac{\ln \hat{\ell}}{\ell}+3 \overline{\mathcal{W}}(\ell)\right]+\mathrm{O}(D-4)
\end{aligned}
$$

The sum of non-singular terms in $\Psi(\ell, \hat{\ell})$ are collected in $\overline{\mathcal{W}}(\ell)$. Its values for $\ell=1,2$ are given in table 10 .

According to (3.61), (3.62) one has

$$
\begin{aligned}
\ddot{g}_{0}(0 ; \ell) & =-\frac{1}{2} \gamma_{1}(\ell)+\frac{1}{4}, \\
\ddot{g}_{1}(0 ; \ell, \hat{\ell}) & =-\mathcal{W}_{1}(\ell, \hat{\ell})=-\tau_{1}(\ell, \hat{\ell})-\frac{1}{16} .
\end{aligned}
$$

\subsection{Checks}

For the special case $\ell=\hat{\ell}=1$

$$
\int_{V_{0}} G(x)^{2} \ddot{g}(x)=\frac{1}{D} \int_{V_{0}} G(x)^{2} .
$$

The lhs. is given by

$$
\Psi_{1}+\Psi_{3}+\Psi_{4}=-\frac{1}{32 \pi^{2}(D-4)}+0.00039168116473927 .
$$

Using (3.74) the rhs. is

$$
\frac{1}{D} \int_{V_{0}} G(x)^{2}=-\frac{1}{32 \pi^{2}(D-4)}+0.00039168116473921,
$$

which agrees with (4.40) up to the 14th digit.

The other contribution is

$$
\int_{V_{0}} G(x)^{2} \ddot{\Delta}(x)=\Psi_{2}+\Psi_{5}+\Psi_{6} .
$$

Since $\square \Delta(x)=-\delta(x)$ and using $\Delta(0)=0$ one has

$$
\int_{V_{0}} G(x)^{2} \ddot{\Delta}(x)=-\frac{1}{D} G(0)^{2}=-\frac{1}{D} g(0)^{2},
$$

in agreement with (4.42).

Also we checked the results for arbitrary $\ell$ by computing $\Psi$ in a completely independent way outlined in appendix B. Doing this we obtained $\overline{\mathcal{W}}(\ell=1)=0.925362611856$, and $\overline{\mathcal{W}}(\ell=2)=0.154824638695$. They differ from the results in table 10 in the 14 th and 7 th

\footnotetext{
${ }^{9}$ For a general shape one should replace here $1 / \ell$ by $1 / \prod_{\mu=0}^{d_{s}} \ell_{\mu}=1 / \mathcal{V}$.
} 


\begin{tabular}{|c|c|c|}
\hline integral & $\ell=1$ & $\ell=2$ \\
\hline$\Psi_{1}$ & 0.002972619688606468 & -0.0002756518941414777 \\
$\Psi_{2 a}$ & -0.0049323221150938617502 & -0.0008736439288408174456 \\
$\Psi_{2 b}$ & 0 & 0.001457734623477771 \\
$\Psi_{3 a}$ & -0.007626138318340436 & 0.004331672346637381 \\
$\Psi_{3 b}$ & 0.00132107926348728 & -0.00183077295570103 \\
$\Psi_{4 b}$ & 0 & 0.0002857740147852 \\
$\Psi_{4 c}$ & 0.0029325487837802 & -0.0036720333298669 \\
$\Psi_{5 d}$ & 0 & -0.000646088213001554 \\
$\psi_{5}$ & 0 & -0.0019520367762068 \\
$\Psi_{7}$ & 0 & 0.002098150204321247 \\
$\mathcal{W}$ & 0.9253626118515132 & 0.1548247146974042 \\
\hline
\end{tabular}

Table 10. Values for $\Psi_{i}$ and $\Psi$ for $\ell=1$ and $\ell=2$.

digits, respectively. We haven't located the source of the discrepancy for $\ell=2$, but the estimate to 7 digits is at present sufficient for our purposes.

Comparing the two approaches, note than in position-space the singularity is only at $x=0$, and several terms considered above have to be treated in DR to cure the singularity. In contrast to this, in the momentum-space approach the singularity of the sunset diagram appears in two loop-momentum variables, hence it is more difficult to handle it.

\subsection{Other results}

$\Psi$ has no pole at $D=3$, and using similar methods for numerical evaluation as described for $D \sim 4$ we obtain ${ }^{10}$ (for $L=L_{s}=1$, cf. (4.1) and (4.36)).

$$
\Psi(\ell)=-\bar{W}(\ell)=-\frac{1}{16 \pi^{2}} \overline{\mathcal{W}}(\ell), \quad d=3,
$$

with

$$
\overline{\mathcal{W}}(\ell)= \begin{cases}2.12506105522294, & \text { for } \ell=1, \\ 1.90198910547056, & \text { for } \ell=2 .\end{cases}
$$

We have also evaluated the sunset integral

$$
\bar{Z}=\int_{x}[G(x)]^{3},
$$

by similar methods.

For $D \sim 3$

$$
\bar{Z} \sim \frac{1}{(4 \pi)^{3}}\left\{-2 \pi\left[\frac{1}{D-3}-2 \ln L\right]+\overline{\mathcal{Z}}(\underline{\ell})\right\}
$$

with

$$
\overline{\mathcal{Z}}= \begin{cases}-10.290523702796, & \text { for } \ell=1, \\ -4.9484964492404, & \text { for } \ell=2 .\end{cases}
$$

\footnotetext{
${ }^{10}$ The notation $\bar{W}$ was used in [1].
} 
Next for $D \sim 4$ :

$$
\bar{Z} \sim \frac{1}{(4 \pi)^{3} L^{2}}\left\{6\left[\frac{1}{D-4}-2 \ln L\right] \mathcal{Z}_{0}(\underline{\ell})+6 \mathcal{Z}_{1}(\underline{\ell}, \hat{\ell})+\overline{\mathcal{Z}}(\underline{\ell})\right\}
$$

where

$$
\begin{gathered}
\mathcal{Z}_{0}(\underline{\ell})=-4 \pi g_{0}(0 ; \underline{\ell})=-\alpha_{1}(\underline{\ell})+1+\frac{1}{\mathcal{V}}, \\
\mathcal{Z}_{1}(\underline{\ell}, \hat{\ell})=-4 \pi g_{1}(0 ; \underline{\ell}, \hat{\ell})=-\rho_{1}(\underline{\ell}, \hat{\ell})-\frac{1}{2}-\frac{\ln (\hat{\ell})}{\mathcal{V}}
\end{gathered}
$$

(cf. (3.59) and (3.60)).

Some numerical values of $\overline{\mathcal{Z}}$ are:

$$
\overline{\mathcal{Z}}_{d=4}= \begin{cases}-2.502240295082, & \text { for } \ell=1, \\ -3.240047780695, & \text { for } \ell=2 .\end{cases}
$$

\section{Dimensionally regularized integrals on a strip}

In our paper [1] we quote the result of a computation of the mass gap of a massless $\mathrm{O}(n)$ sigma model in $3+1$ dimensions with dimensional regularization. The computation involves computing the 2-point function of chiral fields separated in the "time" by distance $t$ in a volume ${ }^{11}$

$$
\Lambda=\left\{x ; x_{0} \in[-T / 2, T / 2], x_{\mu} \in[0, L], \text { for } \mu=1, \ldots, d_{s}, x_{\mu} \in[0, \widehat{L}], \text { for } \mu=d, \ldots, D-1\right\},
$$

with periodic boundary conditions in the $D-1$ "spatial" directions, and free boundary conditions in the time direction (cf. [5]). The mass gap determines the exponential fall off of the 2-point function for $t \rightarrow \infty$ (the limit $T \rightarrow \infty$ being taken first).

The free Green function $G_{F}(x, y)$ is determined by the following four properties:

$$
\begin{aligned}
\int_{x \in \Lambda} G_{F}(x, y) & =0 ; \quad(y \in \bar{\Lambda}) \\
\partial_{0} G_{F}(x, y) & =0 ; \quad\left(x_{0}= \pm T / 2, y \in \bar{\Lambda}\right), \\
G_{F}(x, y) & =G_{F}(y, x) ; \quad(x, y \in \bar{\Lambda}),
\end{aligned}
$$

where $\bar{\Lambda}$ is the interior of $\Lambda$, and for $x, y \in \Lambda$

$$
-\square G_{F}(x, y)=\delta^{(D)}(x-y)-\frac{1}{V_{D}} .
$$

Here the second term is due to the subtraction of the zero mode and

$$
V_{D}=T \bar{V}_{D}, \quad \bar{V}_{D}=L^{d-1} \widehat{L}^{D-d} .
$$

\footnotetext{
${ }^{11}$ Note here $T$ is the extent in the time direction not the temperature.
} 
A representation of the Green function is

$$
\begin{aligned}
G_{F}(x, y)= & \frac{1}{\bar{V}_{D}}\left(-\frac{1}{2}\left|x_{0}-y_{0}\right|+\frac{x_{0}^{2}+y_{0}^{2}}{2 T}+\frac{T}{12}\right) \\
& +\sum_{m=-\infty}^{\infty}\left\{R\left(x_{0}-y_{0}+2 m T, \mathbf{x}-\mathbf{y}\right)+R\left(x_{0}+y_{0}+(2 m+1) T, \mathbf{x}-\mathbf{y}\right)\right\}
\end{aligned}
$$

where

$$
R(z)=\frac{1}{2 \bar{V}_{D}} \sum_{\mathbf{p} \neq 0} \frac{1}{\omega_{\mathbf{p}}} \mathrm{e}^{-\omega_{\mathbf{p}}\left|z_{0}\right|} \mathrm{e}^{i \mathbf{p z}} .
$$

Here the sum goes over $p_{\mu}=2 \pi \nu_{\mu} / L_{\mu}, \mu=1, \ldots, D-1$ with $\nu_{\mu} \in \mathbb{Z}$ and

$$
\omega_{\mathbf{p}}=|\mathbf{p}| .
$$

The function $R(z)$ is defined in (5.8) for all $z \in \mathbb{R}^{D} \backslash 0$; in particular for $\left|z_{0}\right| \rightarrow \infty$ the function $R(z)$ falls exponentially. The singularity at $z=0$ is regularized dimensionally through an alternative representation in terms of the Jacobi theta function (3.52):

$$
R(z)=\frac{1}{4 \pi L^{D-2}} \int_{0}^{\infty} \mathrm{d} u \frac{1}{\sqrt{u}} \mathrm{e}^{-z_{0}^{2} \pi /\left(L^{2} u\right)}\left\{u^{-(D-1) / 2} \prod_{\mu=1}^{D-1} S\left(\frac{\ell_{\mu}^{2}}{u}, \frac{z_{\mu}}{L \ell_{\mu}}\right)-\frac{1}{\mathcal{V}^{\prime}}\right\}
$$

where $\mathcal{V}^{\prime}=\prod_{\mu=1}^{D-1} \ell_{\mu}$.

It turns out that the mass gap to third order PT is completely determined by the following three numbers involving $R: R(0), \ddot{R}(0)$ and $^{12}$

$$
\bar{\Psi}=-L^{2 D-4} W=L^{2 D-4} \int \mathrm{d} y R(y)^{2} \partial_{0}^{2} R(y)
$$

and the rest of this subsection deals with their numerical computation.

First from (5.10) follows

$$
R(0)=\frac{1}{4 \pi L^{D-2}} \int_{0}^{\infty} \mathrm{d} u \frac{1}{\sqrt{u}}\left\{u^{-(D-1) / 2} \prod_{\mu=1}^{D-1} S\left(\frac{\ell_{\mu}^{2}}{u}\right)-\frac{1}{\hat{\ell}^{q}}\right\} .
$$

For numerical evaluation and $\ell_{\mu}=1$ for $\mu=1, \ldots, d_{s}$ and $d>2$ :

$$
R(0)=\frac{1}{4 \pi L^{d-2}}\left\{\int_{0}^{\infty} \frac{\mathrm{d} u}{\sqrt{u}}\left[S(u)^{d-1}\right]_{\mathrm{sub}}+\frac{2}{2-d}-2\right\} .
$$

Using this we find:

$$
\begin{array}{ll}
R(0)=-0.3103732206 \times L^{-1}, & \text { for } d=3, \\
R(0)=-0.2257849594407580334832664917 \times L^{-2}, & \text { for } d=4 .
\end{array}
$$

\footnotetext{
${ }^{12}$ The notation $W=-\int \mathrm{d} y R(y)^{2} \partial_{0}^{2} R(y)$ was used in eq. (5.13) of ref. [1].
} 


\subsection{Calculation of the sunset diagram}

Next we turn to the numerical computation of $\bar{\Psi}$ for $D \sim 4$ in (5.11), setting initially $L=1$ and recovering later the dependence on $L$. In analogy to the computation of $\Psi$ in section 4 it is advantageous to first separate the infinite-volume propagator:

$$
R(z)=\Delta(z)+h(z)
$$

with $^{13}$

$$
h(z)=\frac{1}{4 \pi} \int_{0}^{\infty} \frac{\mathrm{d} u}{\sqrt{u}} \mathrm{e}^{-\pi z_{0}^{2} / u}\left[\frac{1}{u^{(D-1) / 2}} \prod_{\mu=1}^{D-1} S\left(\frac{\ell_{\mu}^{2}}{u}, \frac{z_{\mu}}{\ell_{\mu}}\right)-\frac{1}{\mathcal{V}^{\prime}}-\frac{\mathrm{e}^{-\pi \mathbf{z}^{2} / u}}{u^{(D-1) / 2}}\right] .
$$

We have

$$
\bar{\Psi}=\sum_{r=1}^{7} \bar{\Psi}_{r}
$$

with

$$
\begin{array}{lll}
\bar{\Psi}_{1}=\int_{V_{0}} h(x)^{2} \ddot{h}(x), & \bar{\Psi}_{2}=\int_{V_{0}} \ddot{\Delta}(x) h(x)^{2}, & \bar{\Psi}_{3}=2 \int_{V_{0}} \Delta(x) h(x) \ddot{h}(x), \\
\bar{\Psi}_{4}=\int_{V_{0}} \Delta(x)^{2} \ddot{h}(x), & \bar{\Psi}_{5}=2 \int_{V_{0}} \Delta(x) \ddot{\Delta}(x) h(x), \quad \bar{\Psi}_{6}=\int_{V_{0}} \Delta(x)^{2} \ddot{\Delta}(x), \\
\bar{\Psi}_{7}=\int_{V \backslash V_{0}} R(x)^{2} \ddot{R}(x) . &
\end{array}
$$

Although $h$ is defined for $\ell \rightarrow \infty$, we find it convenient to use (5.16) with a large but finite $\ell$, since the deviation decreases exponentially fast. In this case

$$
\begin{aligned}
& h(x)=g(x)+\frac{1}{\mathcal{V}^{\prime}}\left(\frac{1}{2}\left|x_{0}\right|-\frac{x_{0}^{2}}{2 \ell}-\frac{\ell}{12}\right), \\
& \ddot{h}(x)=\ddot{g}(x)-\frac{1}{\mathcal{V}}+\frac{1}{\mathcal{V}^{\prime}} \delta\left(x_{0}\right),
\end{aligned}
$$

where $\mathcal{V}^{\prime}=\hat{\ell}^{D-4}=1-(D-4) \ln \hat{\ell}+\ldots$. Note that $g(x)$ is a smooth function at $x=0$, hence (5.20) gives explicitly the singular part of $h(x)$ at the origin.

Assuming $\ell \gg 1$ and using the DR rule $\delta^{(D)}(0)=0$ one has

$$
\begin{aligned}
& R(0)=h(0)=g(0)-\frac{\ell}{12}=-0.2257849594407580334832664917, \\
& \ddot{R}(0)=\ddot{h}(0)=\ddot{g}(0)-\frac{1}{\ell}=-0.8375369106960818783868948293 .
\end{aligned}
$$

\footnotetext{
${ }^{13}$ The function $R(z)$ is used in Peter Hasenfratz's rotator paper, the propagator without the contribution of the slow modes, $\mathbf{p}=0$. It is denoted there by $D^{*}(z)$, while our $h(z)$ by $\bar{D}^{*}(z)$; cf. eqs. (49) and (52) of ref. [6].
} 


\subsection{1 $\bar{\Psi}_{1}$}

$$
\bar{\Psi}_{1}=\bar{\Psi}_{1 a}+\bar{\Psi}_{1 b}
$$

where

$$
\bar{\Psi}_{1 a}=\int_{V_{0}} h(x)^{2}[\ddot{g}(x)-1 / \ell]=-0.0119854538
$$

and

$$
\bar{\Psi}_{1 b}=\int_{V_{0}} \delta\left(x_{0}\right) h(x)^{2}=0.0379616384
$$

$\mathrm{So}^{14}$

$$
\bar{\Psi}_{1}=0.0259761846
$$

\subsection{2 $\bar{\Psi}_{2}$}

We split

$$
\bar{\Psi}_{2}=h(0)^{2} \int_{V_{0}} \ddot{\Delta}(x)+\int_{V_{0}} \ddot{\Delta}(x)\left[h(x)^{2}-h(0)^{2}\right] .
$$

The first integral gives

$$
\bar{\Psi}_{2 a}=-h(0)^{2} / 4=-0.0127447119774161875580368870 .
$$

For the second one we divide the volume into 8 pyramids, e.g. the one defined by (4.9) with $\eta \in[0,1 / 2]$ and $u_{i} \in[-1,1]$. The advantage of this is that the Jacobian, $\eta^{3}$ cancels the integrable singularity at $x=0$. Since the integrand is even in all components $x_{\mu}$ one can restrict the integration to $u_{i} \in[0,1]$. Thereby we obtain

$$
\bar{\Psi}_{2 b}=-0.0176501762 \text {. }
$$

Hence

$$
\bar{\Psi}_{2}=-0.0303948882 .
$$

Note that the parameterization (4.9) is also useful to calculate integrals over $V_{0} \backslash S$, taking $1 /\left(2 \sqrt{1+u^{2}}\right) \leq \eta \leq 1 / 2$. The integral over the whole torus can also be done this way.

\subsection{3 $\bar{\Psi}_{3}$}

It is convenient to decompose this into three terms

$$
\bar{\Psi}_{3}=\bar{\Psi}_{3 a}+\bar{\Psi}_{3 b}+\bar{\Psi}_{3 c}
$$

with

$$
\begin{aligned}
\bar{\Psi}_{3 a} & =2 \int_{V_{0}} \Delta(x) \delta\left(x_{0}\right)\left[g(x)-\frac{\ell}{12}\right], \\
\bar{\Psi}_{3 b} & =2 h(0) \ddot{h}(0) \int_{V_{0}} \Delta(x), \\
\bar{\Psi}_{3 c} & =2 \int_{V_{0}} \Delta(x)\left\{\left[\ddot{g}(x)-\frac{1}{\ell}\right] h(x)-h(0) \ddot{h}(0)\right\} .
\end{aligned}
$$

\footnotetext{
${ }^{14}$ We checked that it does not depend on the summation cut, $\alpha$ and $\ell$. Also with numerical differentiation vs. the analytic formula for $\ddot{g}(x)$.
} 
We obtain

$$
\begin{aligned}
& \bar{\Psi}_{3 a}=-0.0810043077, \\
& \bar{\Psi}_{3 b}=-0.04903475008980288 \ddot{h}(0)=0.04106841310696793, \\
& \bar{\Psi}_{3 c}=-0.017070896,
\end{aligned}
$$

where in (5.35) we used (3.73). Collecting contributions

$$
\bar{\Psi}_{3}=-0.0490347500898029 \ddot{h}(0)-0.0980752037=-0.057006791 .
$$

\subsection{4 $\bar{\Psi}_{4}$}

$$
\bar{\Psi}_{4}=\bar{\Psi}_{4 a}+\bar{\Psi}_{4 b}+\bar{\Psi}_{4 c}
$$

with

$$
\begin{aligned}
\bar{\Psi}_{4 a} & =\ddot{h}(0) \Sigma_{1} \\
\bar{\Psi}_{4 b} & =\frac{1}{\mathcal{V}^{\prime}} \int_{V_{0}} \Delta(x)^{2} \delta\left(x_{0}\right), \\
\bar{\Psi}_{4 c} & =\int_{V_{0}} \Delta(x)^{2}[\ddot{g}(x)-\ddot{g}(0)] .
\end{aligned}
$$

Using (3.72)

$$
\begin{aligned}
\bar{\Psi}_{4 a} & =\left[-\frac{1}{8 \pi^{2}(D-4)}+0.011730195135120778\right] \ddot{h}(0) \\
& =-\frac{1}{8 \pi^{2}(D-4)} \ddot{h}(0)-0.0098244713953312651 .
\end{aligned}
$$

For $\bar{\Psi}_{4 b}$ we have

$$
\begin{aligned}
\frac{1}{\mathcal{V}^{\prime}} \int_{S} \Delta(x)^{2} \delta\left(x_{0}\right) & =\frac{1}{\mathcal{V}^{\prime}} A_{D}^{2} \int_{S_{D-1}}|\mathbf{x}|^{-2 D+4}=\frac{1}{\mathcal{V}^{\prime}} A_{D^{2}}^{2} \Omega_{D-1} \int_{0}^{1 / 2} \frac{r^{D-2}}{r^{2 D-4}} \mathrm{~d} r \\
& =\frac{1}{\mathcal{V}^{\prime}} A_{D^{2}}^{2} \Omega_{D-1} \frac{2^{D-3}}{3-D}=\frac{1}{\mathcal{V}^{\prime}}\left(\frac{\Gamma(D / 2-1)}{4 \pi^{D / 2}}\right)^{2} \frac{2 \pi^{(D-1) / 2}}{\Gamma((D-1) / 2)} \frac{2^{D-3}}{3-D} \\
& =-\frac{1}{2 \pi^{3}}+\mathrm{O}(D-4) .
\end{aligned}
$$

Together with

$$
\int_{V_{0} \backslash S} \Delta(x)^{2} \delta\left(x_{0}\right)=0.00272219663411
$$

this gives

$$
\bar{\Psi}_{4 b}=-0.013403570582 .
$$

We evaluate

$$
\bar{\Psi}_{4 c}=0.00028615356 .
$$

Collecting terms one has

$$
\begin{aligned}
\bar{\Psi}_{4} & =\left(-\frac{1}{8 \pi^{2}(D-4)}+0.011730195135120778\right) \ddot{h}(0)-0.01311741702 \\
& =-\frac{1}{8 \pi^{2}(D-4)} \ddot{h}(0)-0.022941888418 .
\end{aligned}
$$

Note that one needs here the expansion of $\ddot{h}(0)$ to $\mathrm{O}(D-4)$. 


\subsection{5 $\bar{\Psi}_{5}$}

Expanding one has

$$
\bar{\Psi}_{5}=\bar{\Psi}_{5 a}+\Psi_{5 b}-\frac{1}{\mathcal{V}} \psi_{5 b}^{(0)}+\Psi_{5 c}+\bar{\Psi}_{5 d}+\bar{\Psi}_{5 e}
$$

with

$$
\begin{aligned}
& \bar{\Psi}_{5 a}=2 h(0) \int_{S} \ddot{\Delta}(x) \Delta(x), \\
& \bar{\Psi}_{5 d}=\frac{1}{\mathcal{V}^{\prime}} \int_{S} \ddot{\Delta}(x) \Delta(x)\left|x_{0}\right|, \\
& \bar{\Psi}_{5 e}=2 \int_{V_{0} \backslash S} \Delta(x) \ddot{\Delta}(x) h(x) .
\end{aligned}
$$

The terms $\Psi_{5 b}, \psi_{5 b}^{(0)}$ and $\Psi_{5 c}$ are given by (4.19), (4.20) and (4.21), respectively.

The term $\bar{\Psi}_{5 a}=0$ since by symmetry it is proportional to $\Delta(0)$. The second and third one are logarithmically divergent and have a pole in $D-4$. Therefore we need here the expansion of $\partial_{\nu}^{2} g(0)$ to first order in $D-4$ given in eqs. (3.61), (3.62) and table 6. The last two integrals are convergent.

$$
\begin{aligned}
\Psi_{5 a}(\ell, \hat{\ell})= & \frac{1}{48 \pi^{2}}\left(\frac{1}{D-4}+\ln 2-\frac{1}{2} \gamma-\frac{1}{2} \ln \pi-\frac{1}{6}\right)\left(\frac{1}{\ell}-4 \ddot{g}(0)\right) \\
& -\frac{1}{48 \pi^{2}}\left(\frac{\ln \hat{\ell}}{\ell}+\frac{1}{4 \ell}\right)+\mathrm{O}(D-4) .
\end{aligned}
$$

Next from (4.28)

$$
\begin{aligned}
-\frac{1}{\mathcal{V}} \psi_{5 b}^{(0)} & =-\frac{1}{\mathcal{V}} \int_{S} \ddot{\Delta}(x) \Delta(x) x_{0}^{2}=\frac{2^{D-5} \Gamma(D / 2-1)(D-1)}{\ell \hat{\ell}^{D-4} \pi^{D / 2} D(D+2)(D-4)} \\
& =\frac{1}{16 \pi^{2} \ell}\left(\frac{1}{D-4}+\ln 2-\frac{1}{2} \gamma-\frac{1}{2} \ln \pi-\ln \hat{\ell}-\frac{1}{12}\right)+\mathrm{O}(D-4) . \\
\bar{\Psi}_{5 d} & =\frac{1}{\mathcal{V}^{\prime}}(D-2) A_{D}^{2} \int_{S} \frac{\left(D t^{2}-r^{2}\right)|t|}{r^{2 D}} \\
& =\frac{1}{\mathcal{V}^{\prime}}(D-2) A_{D}^{2} \Omega_{D} \frac{\rho^{3-D}}{3-D}\left\langle D \cos ^{3} \vartheta-\cos \vartheta\right\rangle
\end{aligned}
$$

where $\rho=1 / 2$ and the averaging is again over $\vartheta \in[0, \pi / 2]$ with weight $\sin ^{D-2} \vartheta$. Using (4.25) we have

$$
\begin{aligned}
\langle\cos \vartheta\rangle & =\frac{\Gamma(D / 2)}{\Gamma(D / 2+1 / 2) \Gamma(1 / 2)}, \\
\left\langle\cos ^{3} \vartheta\right\rangle & =\frac{\Gamma(D / 2)}{\Gamma(D / 2+3 / 2) \Gamma(1 / 2)} .
\end{aligned}
$$

So for $D=4$ we obtain

$$
\begin{aligned}
& \bar{\Psi}_{5 d}=-\frac{2}{5 \pi^{3}}, \\
& \bar{\Psi}_{5 e}=0.00094695753 .
\end{aligned}
$$


Altogether (recalling $\Psi_{5 c}=0$ ):

$$
\bar{\Psi}_{5}=\frac{1}{12 \pi^{2}}\left[-\frac{1}{D-4}+\frac{1}{2} \gamma-\ln 2+\frac{1}{2} \ln \pi+\frac{1}{6}\right] \ddot{h}(0)-0.01195365624 .
$$

\subsection{6 $\bar{\Psi}_{6}$ and $\bar{\Psi}_{7}$}

The integral $\bar{\Psi}_{6}=\Psi_{6}=0$ (see (4.35)), and numerical integration yields

$$
\bar{\Psi}_{7}=0.0000034832546 .
$$

\subsubsection{Final result for $\bar{\Psi}$}

Collecting all terms one obtains the final result ${ }^{15}$

$$
\begin{aligned}
\bar{\Psi} & =-\frac{5}{24 \pi^{2}} \frac{1}{D-4} \ddot{h}(0)-0.0344802923 \ddot{h}(0)-0.1275614973 \\
& =-\frac{5}{24 \pi^{2}} \frac{1}{D-4} \ddot{h}(0)-0.0986829798 .
\end{aligned}
$$

Note that the terms containing $\ell$ cancel and one can take here the $\ell \rightarrow \infty$ limit. This result depends on the $\mathrm{O}(D-4)$ term of $\ddot{h}(0)$ which in turn depends on $\hat{\ell}$, the size of the extra dimensions. However, this dependence cancels from a physical quantity, like the mass gap.

\section{$6 \quad$ Finite volume momentum sums with lattice regularization}

In this section we present results for certain one and two loop momentum sums that we require for our computation of the free energy in massless $\chi$-PT with lattice regularization [1]. We work in an asymmetric $d$-dimensional volume $L_{0}=L_{t}, L_{\mu}=L, \mu=1, \ldots, d_{s}$ and periodic boundary in each direction. We work with the standard lattice action so that the free propagator is given by

$$
G(x)=\frac{1}{V} \sum_{p \neq 0} \frac{\mathrm{e}^{i p x}}{\hat{p}^{2}}
$$

where the sum is over $p_{\mu}=2 \pi n_{\mu} / L_{\mu}, n_{\mu}=0, \ldots, L_{\mu}-1, V=\prod_{\mu=0}^{d_{s}} L_{\mu}$ and $\hat{p}_{\mu}=$ $2 \sin \left(p_{\mu} / 2\right)$. In many equations we will set the lattice spacing $a$ to 1 .

Forward and backward difference operators are defined by

$$
\begin{aligned}
& \partial_{\mu} f(x)=f(x+\hat{\mu})-f(x), \\
& \partial_{\mu}^{*} f(x)=f(x)-f(x-\hat{\mu}),
\end{aligned}
$$

where $\hat{\mu}$ is the unit vector in the $\mu$-direction, and the symmetric derivative

$$
\nabla_{\mu}=\frac{1}{2}\left(\partial_{\mu}+\partial_{\mu}^{*}\right)
$$

\footnotetext{
${ }^{15}$ Eq. (5.61) does not agree with Peter Hasenfratz's result (63) where he has 0.029492025146 instead of 0.0986829798. He also has a $\ln \left(L_{s}\right)$ term, but this is absent in the present convention due to the choice of the scale $L=L_{s}$.
} 
Defining the lattice Laplacian by

$$
\square_{\mu}=\partial_{\mu} \partial_{\mu}^{*}, \quad \square=\sum_{\mu} \square_{\mu},
$$

the propagator (6.1) satisfies

$$
\square G(x)=-\delta(x)+1 / V .
$$

Some useful relations involving the lattice propagator are given in appendix C.

\subsection{Some 1-loop momentum sums}

We define the following 1-loop momentum sums

$$
\begin{aligned}
I_{n m} & =\frac{1}{V} \sum_{p}^{\prime} \frac{\left(\hat{p}_{0}^{2}\right)^{m}}{\left(\hat{p}^{2}\right)^{n}}, \\
J_{n m} & =\frac{1}{V} \sum_{p}^{\prime} \frac{\left(\hat{p}_{0}^{2}\right)^{m} \sum_{\mu} \hat{p}_{\mu}^{4}}{\left(\hat{p}^{2}\right)^{n}}, \\
K_{n m} & =\frac{1}{V} \sum_{p}^{\prime} \frac{\left(\hat{p}_{0}^{2}\right)^{m}\left(\sum_{\mu} \hat{p}_{\mu}^{4}\right)^{2}}{\left(\hat{p}^{2}\right)^{n}}, \\
L_{n m} & =\frac{1}{V} \sum_{p}^{\prime} \frac{\left(\hat{p}_{0}^{2}\right)^{m}}{\left(\hat{p}^{2}\right)^{n} \sum_{\mu \nu} \cos \left(p_{\mu}-p_{\nu}\right) \hat{p}_{\mu}^{2} \hat{p}_{\nu}^{2},} \\
J_{n m k} & =\frac{1}{V} \sum_{p}^{\prime} \frac{\left(\hat{p}_{0}^{2}\right)^{m} \sum_{\mu} \hat{p}_{\mu}^{2 k}}{\left(\hat{p}^{2}\right)^{n}} .
\end{aligned}
$$

Some of these are related to each other e.g.

$$
\begin{aligned}
L_{n m} & =\frac{1}{V} \sum_{p}^{\prime} \frac{\left(\hat{p}_{0}^{2}\right)^{m}}{\left(\hat{p}^{2}\right)^{n}}\left[\left(\sum_{\mu} \hat{p}_{\mu}^{2} \cos p_{\mu}\right)^{2}+\sum_{\mu} \hat{p}_{\mu}^{4} \sin ^{2} p_{\mu}\right] \\
& =I_{n-2, m}-J_{n-1, m}+\frac{1}{4} K_{n m}+J_{n m 3}-\frac{1}{4} J_{n m 4},
\end{aligned}
$$

since $\cos p_{\mu}=1-\frac{1}{2} \hat{p}_{\mu}^{2}$ and $\sin ^{2} p_{\mu}=\hat{p}_{\mu}^{2}-\frac{1}{4} \hat{p}_{\mu}^{4}$.

Note for all dimensions:

$$
\begin{aligned}
& I_{00}=1-a^{d} / V, \\
& I_{01}=2, \\
& I_{02}=6, \\
& I_{10}=G(0), \quad d>2, \\
& I_{20}=\sum_{x} G(x)^{2} .
\end{aligned}
$$




\begin{tabular}{|l|c|}
\hline$n m$ & $I_{n m ; 0}$ \\
\hline 00 & 1 \\
10 & 0.1549333902310602140848372081 \\
11 & 0.25 \\
12 & 0.7066242375215119838793013966 \\
13 & 2.2930387971053356784850269783 \\
14 & 7.9054013578728483268787567644 \\
21 & 0.0387333475577650535212093020 \\
22 & 0.0950666097689397859151627919 \\
23 & 0.2935149141187831114192539608 \\
24 & 0.9869336725760196423390828121 \\
25 & 3.4667969623207723780809806095 \\
32 & 0.0162003867900594714029834178 \\
33 & 0.0436681142558825109634685570 \\
\hline
\end{tabular}

Table 11. Values for $I_{n m ; 0}$ for $d=4$. (For notation cf. (6.19)).

We are interested in the expansion of these sums for $N=L / a \rightarrow \infty$. As has been shown in ref. [7] the 1-loop sums we consider here have a cutoff dependence of the form

$$
A+B \ln N+N^{\delta-d} \sum_{s=0}^{\infty} c_{s} N^{-2 s},
$$

where $\delta$ is determined by behavior of the summand, $|k|^{-\delta}$ at small momenta. The 2loop sums, however, have a more general structure. To cover the different cases we use the notation ${ }^{16}$

$$
X_{A}=\sum_{r}\left(X_{A ; r}+X_{A ; r x} \ln N\right) N^{-r}
$$

\subsubsection{Leading terms}

In many cases the infinite volume limit $L \rightarrow \infty$ of the sums (provided the limit exists) can be computed to arbitrary precision using recursion relations in coordinate space. This observation was first made by Vohwinkel and described in detail for $d=4$ in ref. [8] and later for $d=2$ in [9] and for $d=3$ in [10]. Some results for $d=4$ are given in tables 11, 12 . (According to (6.19) the infinite volume limit is denoted by $I_{n m ; 0}, J_{n m ; 0}$, etc.)

\subsection{Expansion coefficients}

To determine the expansion coefficients of the 1-loop sums we have applied two methods. One is simply to compute the sums to a high precision for a large range of $N$ and fit the data to the expected form, inserting the precisely known leading term when available. Alternatively we obtain the coefficients analytically as integrals involving the theta function (3.52), as described in the next subsection.

\footnotetext{
${ }^{16}$ The 2-loop sum $W_{3 a}$ for $d=2$ given in $(6.102)$ is an exception, it has an extra $\ln ^{2} N /\left(8 \pi^{2}\right)$ term.
} 


\begin{tabular}{|l|c|}
\hline$n m$ & $J_{n m ; 0}$ \\
\hline 10 & 2.8264969500860479355172055864 \\
11 & 6 \\
12 & 18.5673720026100263486623960739 \\
21 & 0.7066242375215119838793013966 \\
22 & 2.1065867439525275504184371975 \\
23 & 7.0275833199331741248758386402 \\
31 & 0.0950666097689397859151627919 \\
32 & 0.2665605938037799596982534915 \\
\hline$n m l$ & $J_{n m l ; 0}$ \\
\hline 213 & 2.2930387971053356784850269783 \\
214 & 7.9054013578728483268787567644 \\
313 & 0.2935149141187831114192539608 \\
314 & 0.9869336725760196423390828121 \\
323 & 0.8658304227039986342356139427 \\
324 & 2.9908252229698117808886771047 \\
\hline$n m$ & $K_{n m ; 0}$ \\
\hline 21 & 18.5673720026100263486623960739 \\
31 & 2.1065867439525275504184371975 \\
32 & 6.4861586895511410207353777372 \\
\hline
\end{tabular}

Table 12. Values for $J_{n m ; 0}, J_{n m l ; 0}, K_{n m ; 0}$ for $d=4$.

\subsubsection{Lattice analogue of the theta function}

Consider the Fourier transform of $f(\theta)$ defined on the interval $0 \leq \theta \leq 2 \pi$ :

$$
\tilde{f}_{m}=\frac{1}{2 \pi} \int_{0}^{2 \pi} f(\theta) \mathrm{e}^{-i m \theta}, \quad f(\theta)=\sum_{m=-\infty}^{\infty} \tilde{f}_{m} \mathrm{e}^{i m \theta} .
$$

Multiplying the equation

$$
\sum_{m=-\infty}^{\infty} \mathrm{e}^{-i m N \theta}=\frac{2 \pi}{N} \sum_{n=-\infty}^{\infty} \delta\left(\theta-\frac{2 \pi}{N} n\right) .
$$

by $(2 \pi)^{-1} f(\theta)$ and integrating over $\theta$ one obtains

$$
\frac{1}{N} \sum_{n=0}^{N-1} f\left(\frac{2 \pi}{N} n\right)=\sum_{m=-\infty}^{\infty} \tilde{f}_{m N} .
$$

Using this relation one obtains

$$
\begin{aligned}
Q_{N}(z)=\frac{1}{N} \sum_{k=0}^{N-1} \exp \left(-z \hat{p}_{k}^{2}\right) & =\mathrm{e}^{-2 z} \sum_{m=-\infty}^{\infty} I_{m N}(2 z) \\
& =\phi_{0}(z)+2 \sum_{m=1}^{\infty} \phi_{m N}(z),
\end{aligned}
$$


where $\hat{p}_{k}=2 \sin (\pi k / N)$ and

$$
\phi_{n}(z)=\mathrm{e}^{-2 z} I_{n}(2 z)
$$

where $I_{n}(z)$ is the modified Bessel function, which for integer $n$ is given by

$$
I_{n}(z)=\frac{1}{\pi} \int_{0}^{\pi} \mathrm{e}^{z \cos \theta} \cos (n \theta) \mathrm{d} \theta .
$$

For convenience of the reader we have summarized some properties of $I_{n}$ that we use in appendix D. For fixed $z, Q_{N}(z)$ approaches $\phi_{0}(z)$ exponentially fast. The approach becomes slower with increasing $z$, but the expansion (D.7) shows that even when the argument increases slower than $N^{2}$ one still has

$$
\lim _{N \rightarrow \infty}\left(Q_{N}\left(c N^{\alpha}\right)-\phi_{0}\left(c N^{\alpha}\right)\right)=0 \quad \text { for } \alpha<2,
$$

with the difference decreasing faster than any inverse power of $N$. This is not true for $z \propto N^{2}$, and for this case one obtains another scaling function.

We introduce the lattice counterpart of $S(x)$ by

$$
\begin{aligned}
S_{N}(x) & =\sum_{k=0}^{N-1} \exp \left(-x N^{2} \hat{p}_{k}^{2} /(4 \pi)\right) \\
& =N \mathrm{e}^{-x N^{2} /(2 \pi)} \sum_{m=-\infty}^{\infty} I_{m N}\left(x N^{2} /(2 \pi)\right) \\
& =N Q_{N}\left(\frac{x N^{2}}{4 \pi}\right)
\end{aligned}
$$

or equivalently

$$
Q_{N}(z)=\frac{1}{N} S_{N}\left(\frac{4 \pi z}{N^{2}}\right)
$$

In the large $N$ limit one has

$$
\lim _{N \rightarrow \infty} S_{N}(x)=S(x) .
$$

Note that the $N \rightarrow \infty$ limit is not uniform in $x$. Since $S_{N}(0)=N$ while $S(x) \sim 1 / \sqrt{x}$ for small $x$, one expects that $S_{N}(x) \approx S(x)$ holds for $N \gg 1 / \sqrt{x}$.

Similarly to the continuum case, the first representation in (6.27) converges very fast for $x \geq 1$ while the second one for $x \leq 1$. In both cases one needs only a few terms in the corresponding sum. For $Q_{N}(z)$ in (6.23) this corresponds to $z>z_{0}(N)$ and $z<z_{0}(N)$ with $z_{0}(N)=N^{2} /(4 \pi)$.

The relatively slowly convergent lattice sums $I_{n m}$ defined in (6.7) can be calculated using $S_{N}(x)$. For $m=0$ one has

$$
\begin{aligned}
I_{n 0} & =\frac{1}{\Gamma(n)} \int_{0}^{\infty} \mathrm{d} z z^{n-1}\left[\prod_{\mu} Q_{N_{\mu}}(z)-\frac{1}{V}\right] \\
& =\left(\frac{N^{2}}{4 \pi}\right)^{n} \frac{1}{\Gamma(n) V} \int_{0}^{\infty} \mathrm{d} x x^{n-1}\left[\prod_{\mu} S_{N_{\mu}}\left(\frac{x}{\ell_{\mu}^{2}}\right)-1\right],
\end{aligned}
$$

where $\ell_{\mu}=N_{\mu} / N$. ( $N$ is arbitrary and one can choose it to be the spatial size, $N=N_{s}$ ). 
For $m>0$

$$
\begin{aligned}
I_{n m} & =\frac{(-1)^{m}}{\Gamma(n)} \int_{0}^{\infty} \mathrm{d} z z^{n-1} Q_{N_{0}}^{(m)}(z) \prod_{\mu \neq 0} Q_{N_{\mu}}(z) \\
& =\frac{\left(N^{2}\right)^{n-m-d / 2}(-1)^{m}}{(4 \pi)^{n-m} \Gamma(n) \ell_{0}^{2 m+1}} \int_{0}^{\infty} \mathrm{d} x x^{n-1} S_{N_{0}}^{(m)}\left(\frac{x}{\ell_{0}^{2}}\right) \prod_{\mu \neq 0} S_{N_{\mu}}\left(\frac{x}{\ell_{\mu}^{2}}\right) .
\end{aligned}
$$

It is useful to split the integral and write (for $\ell_{1}=\ldots=\ell_{d-1}=1, \ell_{0}=\ell$ and general $d$ )

$$
\begin{aligned}
I_{n m}= & \frac{(-1)^{m}}{\Gamma(n)} \int_{0}^{z_{0}} \mathrm{~d} z z^{n-1}\left[Q_{N_{0}}^{(m)}(z) Q_{N}^{d-1}(z)-\frac{\delta_{m 0}}{N^{d} \ell}\right] \\
& +\frac{\left(N^{2}\right)^{n-m-d / 2}(-1)^{m}}{(4 \pi)^{n-m} \Gamma(n) \ell^{2 m+1}} \int_{x_{0}}^{\infty} \mathrm{d} x x^{n-1}\left[S_{N_{0}}^{(m)}\left(\frac{x}{\ell^{2}}\right) S_{N}^{d-1}(x)-\delta_{m 0}\right],
\end{aligned}
$$

where $x_{0}=4 \pi z_{0} / N^{2}$.

To obtain the expansion of $I_{n m}$ for large $N$ we need the asymptotic expansion of $S_{N}(x)(6.33)$ in the next subsection.

\subsubsection{Asymptotic behavior for $N \rightarrow \infty$}

As discussed before, for $z=z_{N} \propto N^{\alpha}$ with $\alpha<2$ the correction term $Q_{N}\left(z_{N}\right)-\phi_{0}\left(z_{N}\right)$ goes to zero exponentially fast for $N \rightarrow \infty$.

Expanding (6.27) in $1 / N^{2}$ one obtains an asymptotic expansion

$$
S_{N}^{\mathrm{as}}(x)=S(x)+\frac{1}{N^{2}} \bar{S}_{1}(x)+\frac{1}{N^{4}} \bar{S}_{2}(x)+\ldots
$$

where

$$
\begin{aligned}
& \bar{S}_{1}(x)=\frac{\pi}{3} x S^{\prime \prime}(x) \\
& \bar{S}_{2}(x)=\frac{\pi^{2}}{90}\left(4 x S^{(3)}(x)+5 x^{2} S^{(4)}(x)\right) \\
& \bar{S}_{3}(x)=\frac{\pi^{3}}{5670}\left(18 x S^{(4)}(x)+84 x^{2} S^{(5)}(x)+35 x^{3} S^{(6)}(x)\right), \\
& \bar{S}_{4}(x)=\frac{\pi^{4}}{340200}\left(48 x S^{(5)}(x)+696 x^{2} S^{(6)}(x)+840 x^{3} S^{(7)}(x)+175 x^{4} S^{(8)}(x)\right) .
\end{aligned}
$$

As mentioned above the expansion (6.33) is not uniform.

We will also need the behavior of the corresponding terms at $x=0$ :

$$
\begin{aligned}
\bar{S}_{1} & \sim \frac{\pi}{4} x^{-3 / 2}, & \bar{S}_{2} & \sim \frac{9 \pi^{2}}{32} x^{-5 / 2}, \\
\bar{S}_{3} & \sim \frac{75 \pi^{3}}{128} x^{-7 / 2}, & \bar{S}_{4} & \sim \frac{3675 \pi^{4}}{2048} x^{-9 / 2} .
\end{aligned}
$$

Introducing $z=y N^{\alpha}$ with $0<\alpha<2$ one has

$$
S_{N}^{\mathrm{as}}\left(4 \pi N^{\alpha-2} y\right) \sim N \phi_{0}\left(N^{\alpha} y\right)
$$


This means that the singularity of $S_{N}^{\text {as }}(x)$ at $x \sim 0$ matches the asymptotic behavior of $\phi_{0}(z)=\mathrm{e}^{-2 z} I_{0}(2 z)$ for $z \rightarrow \infty$. The relation can be checked using the asymptotic form (D.6) and $S(x)=x^{-1 / 2}\left[1+\mathrm{O}\left(\mathrm{e}^{-\pi / x}\right)\right]$.

The relation between the $x>1$ and $x<1$ regions for $S(x)$ is given in (2.23). Differentiating it one obtains the corresponding relations between the derivatives of $S(x)$.

At $x=x_{0}(N)$ the difference $S_{N}(x)-S(x)$ changes sign: for $x<x_{0}(N)$ one has $S_{N}(x)<S(x)$ while for $x>x_{0}(N)$ one has $S_{N}(x)>S(x)$. Interestingly, for $N \geq 8$ one has with a very good precision $x_{0}(N) \approx 4 \pi z_{0} / N^{2}$ where $z_{0}=0.06447351504$.

For $N \rightarrow \infty, N_{0}=\ell N$ in $d$-dimensions one has for the bracket appearing in the integrand of $(6.32)$

$$
\begin{aligned}
& S_{N_{0}}^{(m)}\left(\frac{x}{\ell^{2}}\right) S_{N}^{d-1}(x)-\delta_{m 0} \\
& \quad \sim \Psi_{m}(x ; \ell)=\Phi_{0 m}(x ; \ell)+\frac{1}{N^{2}} \Phi_{1 m}(x ; \ell)+\frac{1}{N^{4}} \Phi_{2 m}(x ; \ell)+\ldots,
\end{aligned}
$$

where

$$
\begin{aligned}
\Phi_{0 m}(x ; \ell)= & S^{d-1}(x) S^{(m)}\left(\frac{x}{\ell^{2}}\right)-\delta_{m 0}, \\
\Phi_{1 m}(x ; \ell)= & S^{d-1}(x) \bar{S}_{1}^{(m)}\left(\frac{x}{\ell^{2}}\right) \frac{1}{\ell^{2}}+(d-1) S^{d-2}(x) \bar{S}_{1}(x) S^{(m)}\left(\frac{x}{\ell^{2}}\right), \\
\Phi_{2 m}(x ; \ell)= & S^{d-1}(x) \bar{S}_{2}^{(m)}\left(\frac{x}{\ell^{2}}\right) \frac{1}{\ell^{4}}+(d-1) S^{d-2}(x) \bar{S}_{1}(x) \bar{S}_{1}^{(m)}\left(\frac{x}{\ell^{2}}\right) \frac{1}{\ell^{2}} \\
& +(d-1) S^{d-2}(x) \bar{S}_{2}(x) S^{(m)}\left(\frac{x}{\ell^{2}}\right)+\frac{1}{2}(d-1)(d-2) S^{d-3}(x) \bar{S}_{1}^{2}(x) S^{(m)}\left(\frac{x}{\ell^{2}}\right) .
\end{aligned}
$$

Their leading singularity for $x \rightarrow 0$ is given by

$$
\begin{aligned}
\Phi_{0 m}^{\text {sing }}(x ; \ell)= & (-1)^{m} \frac{\Gamma(m+1 / 2)}{\Gamma(1 / 2)} \ell^{2 m+1} x^{-(m+d / 2)}, \\
\Phi_{1 m}^{\text {sing }}(x ; \ell)= & (-1)^{m} \frac{\pi}{4} \frac{\Gamma(m+1 / 2)}{\Gamma(1 / 2)}(2 m+d) \ell^{2 m+1} x^{-(m+1+d / 2)}, \\
\Phi_{2 m}^{\text {sing }}(x ; \ell)= & (-1)^{m} \frac{\pi^{2}}{32} \frac{\Gamma(m+1 / 2)}{\Gamma(1 / 2)}\left(12 m^{2}+20 m+4 d m+8 d+d^{2}\right) \\
& \times \ell^{2 m+1} x^{-(m+2+d / 2)} .
\end{aligned}
$$

As illustrated in the next subsection, for the case when $I_{n m}$ has a finite $N \rightarrow \infty$ limit (i.e. $2(n-m)<d)$ one obtains ${ }^{17}$

$$
I_{n m}=\frac{(-1)^{m} N^{2(n-m)-d}}{(4 \pi)^{n-m} \Gamma(n) \ell^{2 m+1}} \int_{0}^{\infty} \mathrm{d} x x^{n-1}\left[\Psi_{m}(x ; \ell)-\Psi_{m}^{\text {sing }}(x ; \ell)\right] .
$$

Expanding in powers of $1 / N^{2}$ one gets for the coefficient of $N^{-\nu}$

$$
I_{n m ; \nu}=\frac{(-1)^{m}}{(4 \pi)^{n-m} \Gamma(n) \ell^{2 m+1}} \int_{0}^{\infty} \mathrm{d} x x^{n-1}\left[\Phi_{k m}(x ; \ell)-\Phi_{k m}^{\text {sing }}(x ; \ell)\right],
$$

where $k=0,1, \ldots$ and $\nu=2(k+m-n)-d$.

\footnotetext{
${ }^{17}$ Eqs. (6.44), (6.45) give the correct answer for some higher coefficients even when $2(n-m) \geq d$, but these cases need a special treatment, like for $I_{20}$ in $d=4$ discussed below.
} 


\subsubsection{Examples: $I_{10}, I_{21}$ for $d>2$}

Consider $I_{10}$ for $d>2$ in a $N_{0} \times N^{d_{s}}$ volume $\left(N_{0}=L_{0} / a=N \ell, N=L_{s} / a\right)$ :

$$
\begin{aligned}
I_{10} & =\int_{0}^{\infty} \mathrm{d} z\left[Q_{N \ell}(z) Q_{N}(z)^{d_{s}}-\frac{1}{N^{d} \ell}\right] \\
& =\int_{0}^{z_{0}} \mathrm{~d} z Q_{N \ell}(z) Q_{N}(z)^{d_{s}}-\frac{z_{0}}{N^{d} \ell}+\int_{z_{0}}^{\infty} \mathrm{d} z\left[Q_{N \ell}(z) Q_{N}(z)^{d_{s}}-\frac{1}{N^{d} \ell}\right] .
\end{aligned}
$$

One can show that for $z_{0} \propto N^{2}$ only the first term contributes to the constant piece $I_{10 ; 0}$ and one obtains ${ }^{18}$

$$
I_{10 ; 0}=\int_{0}^{\infty} \mathrm{d} z\left[\phi_{0}(z)\right]^{d} .
$$

To calculate the $1 / N^{r}$ corrections we consider the differences

$$
\Delta_{A}\left(N, z_{0}\right)=\int_{0}^{z_{0}} \mathrm{~d} z\left[Q_{N \ell}(z) Q_{N}(z)^{d_{s}}-\phi_{0}(z)^{d}\right]
$$

and

$$
\Delta_{B}\left(N, z_{0}\right)=\int_{z_{0}}^{\infty} \mathrm{d} z\left[Q_{N \ell}(z) Q_{N}(z)^{d_{s}}-Q_{N \ell}^{\mathrm{as}}(z) Q_{N}^{\mathrm{as}}(z)^{d_{s}}\right]
$$

where

$$
Q_{N}^{\mathrm{as}}(z)=\frac{1}{N} S_{N}^{\mathrm{as}}\left(\frac{4 \pi z}{N^{2}}\right) .
$$

For $z_{0}=z_{0}(N)=c N^{\alpha}$, where $1<\alpha<2$ both integrals (6.48) and (6.49) go to zero for $N \rightarrow \infty$ exponentially fast.

We have

$$
\begin{aligned}
I_{10}-I_{10 ; 0}= & -\int_{z_{0}}^{\infty} \mathrm{d} z \phi_{0}^{d}(z)-\frac{z_{0}}{N^{d} \ell}+\int_{z_{0}}^{\infty} \mathrm{d} z\left[Q_{N \ell}^{\mathrm{as}}(z) Q_{N}^{\mathrm{as}}(z)^{d_{s}}-\frac{1}{N^{d} \ell}\right] \\
& +\Delta_{A}\left(N, z_{0}\right)+\Delta_{B}\left(N, z_{0}\right) .
\end{aligned}
$$

Here the $z_{0}$ dependence should cancel, i.e. $Q_{N}^{\text {as }}\left(z_{0}\right)$ and $\phi_{0}\left(z_{0}\right)$ should have the same asymptotic behavior for $z_{0}(N)=c N^{\alpha}$ and $N \rightarrow \infty$. Note that the argument $x_{0}(N)=$ $4 \pi z_{0}(N) / N^{2} \propto N^{\alpha-2}$ of the functions $S(x)$ in (6.50) goes to zero in this limit. Hence the contributions from the large- $z$ asymptotic of $\phi_{0}(z)$ and the small- $x$ asymptotic of $S_{N}^{\text {as }}(x) / N$ cancel each other. This is indeed the case, one has ${ }^{19}$

$$
\frac{1}{N} S_{N}^{\text {as }}\left(\frac{4 \pi z_{0}}{N^{2}}\right) \sim \phi_{0}\left(z_{0}\right) \sim \frac{1}{\sqrt{4 \pi z_{0}}}\left(1+\frac{1}{16 z_{0}}+\frac{9}{512 z_{0}^{2}}+\ldots\right) .
$$

With $x_{0}=4 \pi z_{0} / N^{2}$ one has

$$
\begin{aligned}
& \int_{z_{0}}^{\infty} \mathrm{d} z\left[Q_{N \ell}^{\mathrm{as}}(z) Q_{N}^{\mathrm{as}}(z)^{d_{s}}-\frac{1}{N^{d} \ell}\right]=\frac{1}{4 \pi N^{d-2} \ell} \int_{x_{0}}^{\infty} \mathrm{d} x\left[S_{N \ell}^{\mathrm{as}}\left(\frac{x}{\ell^{2}}\right) S_{N}^{\mathrm{as}}(x)^{d_{s}}-1\right] \\
& =\frac{1}{4 \pi N^{d-2} \ell} \int_{x_{0}}^{\infty} \mathrm{d} x\left\{\Phi_{00}(x ; \ell)+\frac{1}{N^{2}} \Phi_{10}(x ; \ell)+\frac{1}{N^{4}} \Phi_{20}(x ; \ell)+\ldots\right\} .
\end{aligned}
$$

\footnotetext{
${ }^{18}$ The result of numerical integration with MAPLE for $d=4$ agrees to 27 digits with the exact value given in table 11.

${ }^{19}$ The $N$-dependence on $Q_{N}^{\text {as }}(z)$ cancels in the asymptotic expansion.
} 
The subtraction of the integral of $\phi_{0}(z)^{d}$ amounts to subtracting from each term its singular part for $x \rightarrow 0$. So finally we have for $d>2$

$$
I_{10}=I_{10 ; 0}+\frac{1}{N^{d-2}} I_{10 ; d-2}+\frac{1}{N^{d}} I_{10 ; d}+\frac{1}{N^{d+2}} I_{10 ; d+2}+\ldots
$$

where

$$
\begin{aligned}
I_{10 ; d-2} & =\frac{1}{4 \pi \ell} \int_{0}^{\infty} \mathrm{d} x\left[\Phi_{00}(x ; \ell)-\frac{\ell}{x^{d / 2}}\right], \\
I_{10 ; d} & =\frac{1}{4 \pi \ell} \int_{0}^{\infty} \mathrm{d} x\left[\Phi_{10}(x ; \ell)-\frac{\pi d \ell}{4 x^{d / 2+1}}\right], \\
I_{10 ; d+2} & =\frac{1}{4 \pi \ell} \int_{0}^{\infty} \mathrm{d} x\left[\Phi_{20}(x ; \ell)-\frac{\pi^{2} \ell d(d+8)}{32 x^{d / 2+2}}\right] .
\end{aligned}
$$

Comparing (6.55) with $\beta_{1}$ given in (2.42) and (2.46) for $d=3,4$ we obtain

$$
I_{10 ; d-2}=-\beta_{1}, \quad \text { for } d=3,4,
$$

relating coefficients of the lattice expansion to shape coefficients in DR. This is just one example of many such relations.

Repeating the steps used above one gets for the expansion coefficients of $I_{21}$ :

$$
I_{21}=\frac{1}{d} I_{10 ; 0}+\frac{1}{N^{d-2}} I_{21 ; d-2}+\frac{1}{N^{d}} I_{21 ; d}+\ldots, \quad d>2,
$$

with

$$
I_{21 ; d-2}=-\frac{1}{4 \pi \ell^{3}} \int_{0}^{\infty} \mathrm{d} x x\left[\Phi_{01}(x ; \ell)+\frac{\ell^{3}}{2 x^{(d+2) / 2}}\right] .
$$

Again, for $d=3,4 I_{21 ; d-2}$ is related to the corresponding DR shape coefficient e.g.

$$
I_{21 ; 2}=\frac{1}{8 \pi}\left(\gamma_{2}-1\right)=L^{2} \bar{I}_{21}, \quad d=4
$$

Next

$$
I_{21 ; d}=-\frac{1}{4 \pi \ell^{3}} \int_{0}^{\infty} \mathrm{d} x x\left[\Phi_{11}(x ; \ell)+\frac{(d+2) \pi \ell^{3}}{8 x^{(d+4) / 2}}\right] .
$$

So far in this subsection we have only considered sums which have a finite infinite volume limit. As an example of a sum which does not have this property we consider $I_{20}$. For $d=3 I_{20}$ is linearly divergent (see (6.74), (6.75)).

In the rest of this subsection we only consider the case $d=4$ for which $I_{20}$ is logarithmically divergent for $N \rightarrow \infty$. Here we will separate the $\sim \log (N)$ and the constant terms. ${ }^{20}$ Restricting also to $\ell=1$ we have

$$
I_{20}=\int_{0}^{z_{0}} \mathrm{~d} z z\left(Q_{N}^{4}(z)-\frac{1}{N^{4}}\right)+\frac{1}{16 \pi^{2}} \int_{x_{0}}^{\infty} \mathrm{d} x x\left(S_{N}^{4}(x)-1\right)
$$

where $x_{0}=4 \pi z_{0} / N^{2}$. Choosing $z_{0}=c N^{2-\epsilon}$ with some fixed small $\epsilon>0$ in the first term one could replace $Q_{N}(z)$ by $\phi_{0}(z)$ up to exponentially small corrections.

\footnotetext{
${ }^{20}$ For our work in ref. [1] the constant term is actually needed only for the renormalization.
} 
One has

$$
\begin{aligned}
I_{20}^{A}= & \int_{0}^{z_{0}} \mathrm{~d} z z\left(Q_{N}^{4}(z)-\frac{1}{N^{4}}\right) \sim \int_{0}^{z_{0}} \mathrm{~d} z z \phi_{0}^{4}(z)-\frac{z_{0}^{2}}{2 N^{4}} \\
= & \int_{0}^{1} \mathrm{~d} z z \phi_{0}^{4}(z)+\int_{1}^{z_{0}} \mathrm{~d} z z\left(\phi_{0}^{4}(z)-\frac{1}{16 \pi^{2} z^{2}}\right) \\
& +\frac{1}{16 \pi^{2}} \log z_{0}-\frac{z_{0}^{2}}{2 N^{4}} .
\end{aligned}
$$

In the second integral of (6.63) one can replace $S_{N}(x)$ by $S(x)$ up to O $\left(1 / N^{2}\right)$ correction.

$$
\begin{aligned}
I_{20}^{B}= & \frac{1}{16 \pi^{2}} \int_{x_{0}}^{\infty} \mathrm{d} x x\left(S_{N}^{4}(x)-1\right) \sim \frac{1}{16 \pi^{2}} \int_{x_{0}}^{\infty} \mathrm{d} x x\left(S^{4}(x)-1\right) \\
= & \frac{1}{16 \pi^{2}} \int_{x_{0}}^{1} \mathrm{~d} x x\left(S^{4}(x)-\frac{1}{x^{2}}\right)+\frac{1}{16 \pi^{2}} \int_{1}^{\infty} \mathrm{d} x x\left(S^{4}(x)-1\right) \\
& -\frac{1-x_{0}^{2}}{32 \pi^{2}}-\frac{1}{16 \pi^{2}} \log x_{0} .
\end{aligned}
$$

Adding the two terms and for large $N$ one obtains

$$
\begin{aligned}
I_{20}= & \int_{0}^{1} \mathrm{~d} z z \phi_{0}^{4}(z)+\int_{0}^{\infty} \mathrm{d} z z\left(\phi_{0}^{4}(z)-\frac{1}{16 \pi^{2} z^{2}}\right) \\
& +\frac{1}{16 \pi^{2}} \int_{0}^{1} \mathrm{~d} x x\left(S^{4}(x)-\frac{1}{x^{2}}\right)+\frac{1}{16 \pi^{2}} \int_{1}^{\infty} \mathrm{d} x x\left(S^{4}(x)-1\right) \\
& -\frac{1}{32 \pi^{2}}+\frac{1}{16 \pi^{2}} \log \left(\frac{N^{2}}{4 \pi}\right)+\mathrm{O}\left(1 / N^{2}\right) .
\end{aligned}
$$

Evaluating the integrals one has

$$
I_{20}=\frac{1}{8 \pi^{2}} \log N+0.01004098140549470847620108+\mathrm{O}\left(1 / N^{2}\right)
$$

The coefficients $I_{20 ; 2}$ and $I_{20 ; 4}$ for $d=4$ are given in the next subsection.

One can repeat these steps for the general case of $I_{n m}$. Using the representation (6.32) one reproduces the form of the expansion given by (6.18). The $\log N$ term comes from the $1 / x$ and $1 / z$ terms of the corresponding integrands while in the rest one can set $x_{0}=0$ and $z_{0}=\infty$, as in (6.64) and (6.65). Finally, the coefficient $N^{2(n-m)-d}$ in front of the second integral in (6.32) reproduces the prefactor $N^{\delta-d}$ of the sum in (6.18). 


\subsubsection{Expansions for $d=4$}

We are interested in the expansion of the lattice sums for $N^{-1}=a / L \rightarrow 0$ (at a fixed aspect ratio $\ell$ ) up to and including the $\mathrm{O}\left(a^{4} / L^{4}\right)$ terms. For the sums we require we have:

$$
\begin{aligned}
& I_{00}=1-\ell^{-1} N^{-4} \\
& I_{10}=I_{10 ; 0}+I_{10 ; 2} N^{-2}+I_{10 ; 4} N^{-4}+\ldots \\
& I_{11}=I_{11 ; 0}+I_{11 ; 4} N^{-4} \\
& I_{20}=\ln N /\left(8 \pi^{2}\right)+I_{20 ; 0}+I_{20 ; 2} N^{-2}+I_{20 ; 4} N^{-4}+\ldots \\
& I_{21}=I_{21 ; 0}+I_{21 ; 2} N^{-2}+I_{21 ; 4} N^{-4}+\ldots \\
& I_{22}=I_{22 ; 0}+I_{22 ; 4} N^{-4}+\ldots \\
& I_{31}=\ln N /\left(32 \pi^{2}\right)+I_{31 ; 0}+I_{31 ; 2} N^{-2}+I_{31 ; 4} N^{-4}+\ldots \\
& I_{32}=I_{32 ; 0}+I_{32 ; 2} N^{-2}+I_{32 ; 4} N^{-4}+\ldots \\
& I_{33}=I_{33 ; 0}+I_{33 ; 4} N^{-4}+\ldots \\
& J_{31}=J_{31 ; 0}+J_{31 ; 4} N^{-4}+\ldots
\end{aligned}
$$

The coefficients $I_{n m ; \nu}$ can be calculated (at least for the cases with finite infinite-volume limit) from (6.45).

Next consider the lattice sum $J_{31}$ (see (6.8)); for $d=4$ one has

$$
J_{31}=\frac{1}{V} \sum_{p}^{\prime} \frac{\left(\hat{p}_{0}^{2}\right)^{3}}{\left(\hat{p}^{2}\right)^{3}}+J_{31}^{B},
$$

with

$$
J_{31}^{B}=\frac{3}{V} \sum_{p}^{\prime} \frac{\hat{p}_{0}^{2}\left(\hat{p}_{1}^{2}\right)^{2}}{\left(\hat{p}^{2}\right)^{3}} .
$$

The first term in (6.69) is $I_{33}$, hence

$$
J_{31 ; 4}=I_{33 ; 4}+J_{31 ; 4}^{B}, \text { for } d=4,
$$

with

$$
J_{31 ; 4}^{B}=-\frac{3}{2 \ell^{3}} \int_{0}^{\infty} \mathrm{d} x x^{2}\left[S(x)^{2} S^{\prime \prime}(x) S^{\prime}\left(\frac{x}{\ell^{2}}\right)+\frac{3 \ell^{3}}{8 x^{5}}\right]
$$

In tables 13 and 14 we give values of the coefficients above for $\ell=1,2,3$ and $\ell=4,5$ respectively using the integral representation (6.45) with MAPLE. We checked that all results agreed to at least 12 digits in all cases with fits of the data (using the precise infinite volume results when available). 


\begin{tabular}{|c|r|r|c|}
\hline & $\ell=1$ & $\ell=2$ & $\ell=3$ \\
\hline$I_{10 ; 2}$ & -0.1404609855453658 & -0.0591149364827813 & 0.0242150467817181 \\
$I_{10 ; 4}$ & 0.1418858055568331 & 0.1445281475197173 & 0.1582885920258339 \\
$I_{11 ; 4}$ & -0.25 & 0.3374033678947278 & 0.5042033315676824 \\
$I_{20 ; 2}$ & 0.0014757515175671 & 0.0122627013456392 & 0.0261308665866801 \\
$I_{20 ; 4}$ & 0.0731806946434512 & 0.0593077967014635 & 0.0615529993132684 \\
$I_{21 ; 2}$ & -0.0351152463863414 & 0.0537547083123951 & 0.1371074647314091 \\
$I_{21 ; 4}$ & 0.0354714513892083 & 0.1554316713871277 & 0.1699444287054589 \\
$I_{22 ; 4}$ & 0.4256574166704993 & 0.7569495889658734 & 0.9229739816883220 \\
$I_{31 ; 2}$ & 0.0003689378793918 & 0.0167916533065880 & 0.0307265836535553 \\
$I_{31 ; 4}$ & 0.0182951736608628 & 0.0404550162559959 & 0.0435488531765849 \\
$I_{32 ; 2}$ & 0.0022136272763506 & 0.0820388915996510 & 0.1653308457382867 \\
$I_{32 ; 4}$ & 0.1647046454257431 & 0.1861803595341033 & 0.1990444597971972 \\
$I_{33 ; 4}$ & 0.5483743850461059 & 1.0693538988138826 & 1.2370421989724505 \\
$J_{31 ; 4}$ & 0.4256574166704993 & 1.3110648228057027 & 1.4811535630769142 \\
\hline
\end{tabular}

Table 13. Values for $I_{m n ; 2}, I_{m n ; 4}, J_{31 ; 4}$ for $d=4$ and $\ell=1,2,3$.

\begin{tabular}{|c|c|c|}
\hline & $\ell=4$ & $\ell=5$ \\
\hline$I_{10 ; 2}$ & 0.1075483739041892 & 0.1908817072259303 \\
$I_{10 ; 4}$ & 0.1652326717601757 & 0.1693993375068985 \\
$I_{11 ; 4}$ & 0.5875369102375629 & 0.6375369106952257 \\
$I_{20 ; 2}$ & 0.0400196677374950 & 0.0539085563322884 \\
$I_{20 ; 4}$ & 0.0628240364390694 & 0.0635879153141902 \\
$I_{21 ; 2}$ & 0.2204408534728138 & 0.3037741869459578 \\
$I_{21 ; 4}$ & 0.1768917723653977 & 0.1810584492251718 \\
$I_{22 ; 4}$ & 1.0063053711184669 & 1.0563053660562876 \\
$I_{31 ; 2}$ & 0.0446158019765458 & 0.0585046924277057 \\
$I_{31 ; 4}$ & 0.0448289656504658 & 0.0455929029967931 \\
$I_{32 ; 2}$ & 0.2486639742820079 & 0.3319973068786403 \\
$I_{32 ; 4}$ & 0.2059796377872626 & 0.2101462558490117 \\
$I_{33 ; 4}$ & 1.3203816834539149 & 1.3703817074781796 \\
$J_{31 ; 4}$ & 1.5645033197516197 & 1.6145033783791748 \\
\hline
\end{tabular}

Table 14. Values for $I_{m n ; 2}, I_{m n ; 4}, J_{31 ; 4}$ for $d=4$ and $\ell=4,5$. 


\subsubsection{Expansions for $d=3$}

Some infinite volume values are

$$
\begin{aligned}
& I_{10 ; 0}=0.252731009859, \\
& I_{12 ; 0}=0.913649942701, \\
& I_{21 ; 0}=0.084243669953, \\
& I_{22 ; 0}=0.164845993428, \\
& I_{23 ; 0}=0.486683859112, \\
& I_{32 ; 0}=0.046176554504, \\
& I_{33 ; 0}=0.096821201585 .
\end{aligned}
$$

Also in this case we determined the coefficients both by fitting the $N$-dependence and by direct calculation using (6.45), when it was applicable. In all cases we got an agreement within the precision of the fitting procedure. (The worst case was that of $I_{30 ; 1}$ where the fit gave only four significant digits.)

For $\ell=1$ :

$$
\begin{aligned}
I_{10}= & I_{10 ; 0}-0.225784959441 N^{-1}+0.0428997562958 N^{-3}+\ldots, \\
I_{11}= & \frac{1}{3}-\frac{1}{3} N^{-3}, \\
I_{12}= & I_{12 ; 0}+\ldots, \\
I_{20}= & 0.010607528892 N+0.012164158583-0.0155358881130 N^{-1}+\ldots, \\
I_{21}= & I_{21 ; 0}-0.0752616531469 N^{-1}+0.014299918765276429 N^{-3}+\ldots, \\
I_{22}= & I_{22 ; 0}+0.171599025183 N^{-3}+\ldots, \\
I_{23}= & I_{23 ; 0}+\ldots, \\
I_{30}= & 0.000136552463 N^{3}+0.00191507947957 N+0.000837762 \\
& -0.000388292243730 N^{-1}+\ldots, \\
I_{31}= & 0.00353584296399 N+0.004054719528-0.00517862937101 N^{-1}+\ldots, \\
I_{32}= & I_{32 ; 0}-0.0310717762260 N^{-1}+0.0973259651475 N^{-3}+\ldots, \\
I_{33}= & I_{33 ; 0}+0.348442731224 N^{-3}+\ldots
\end{aligned}
$$

For $\ell=2$ :

$$
\begin{aligned}
& I_{10}=I_{10 ; 0}-0.143704325288 N^{-1}+0.0614336837790 N^{-3}+\ldots, \\
& I_{11}=\frac{1}{3}+0.218784444721 N^{-3}+\ldots, \\
& I_{12}=I_{12 ; 0}+\ldots, \\
& I_{20}=0.020216123622 N+0.012164158583-0.00356885724612 N^{-1}+\ldots, \\
& I_{21}=I_{21 ; 0}+0.0114671446126 N^{-1}+0.0992873225148 N^{-3}+\ldots, \\
& I_{22}=I_{22 ; 0}+0.578733423280 N^{-3}+\ldots, \\
& I_{23}=I_{23 ; 0}+\ldots,
\end{aligned}
$$




$$
\begin{aligned}
I_{30}= & 0.001155699930 N^{3}+0.00424327053578 N+0.000837763 \\
& +0.00161399011894 N^{-1}+\ldots, \\
I_{31}= & 0.0133862498578 N+0.004054719527+0.0102078527937 N^{-1}+\ldots, \\
I_{32}= & I_{32 ; 0}+0.0503041764078 N^{-1}+0.132427332872 N^{-3}+\ldots, \\
I_{33}= & I_{33 ; 0}+0.847216528219 N^{-3}+\ldots
\end{aligned}
$$

Here only $I_{20}, I_{30}$ and $I_{31}$ diverge for $N \rightarrow \infty$. However, also in these cases the coefficients $I_{20 ; 1}, I_{30 ;-1}, I_{30 ; 1}, I_{31 ;-1}, I_{31 ; 1}$ are correctly given by (6.45).

The shape coefficients $I_{10 ; 1}$ and $I_{20 ;-1}$ are related to $\beta_{n}$ defined in (2.42) through:

$$
\begin{aligned}
I_{10 ; 1} & =-\beta_{1}, \\
I_{20 ;-1} & =\beta_{2} .
\end{aligned}
$$

Further there are relations to $\widetilde{\beta}_{n}$ defined in [11]:

$$
\begin{aligned}
I_{21 ; 1} & =-\frac{1}{6} \widetilde{\beta}_{1}, \\
I_{31 ;-1} & =\frac{1}{12} \widetilde{\beta}_{2} .
\end{aligned}
$$

\subsubsection{Expansions for $d=2$}

In this subsection we consider only the $\ell=1$ case and concentrate on terms which do not vanish in the infinite volume limit.

For the logarithmically divergent sum $I_{10}$ we have

$$
I_{10}=\int_{0}^{z_{0}} \mathrm{~d} z\left(Q_{N}^{2}(z)-\frac{1}{N^{2}}\right)+\frac{1}{4 \pi} \int_{x_{0}}^{\infty} \mathrm{d} x\left(S_{N}^{2}(x)-1\right)
$$

where $x_{0}=4 \pi z_{0} / N^{2}$. Choosing $z_{0}=c N^{2-\epsilon}$ with some fixed small $\epsilon>0$ in the first term one could replace $Q_{N}(z)$ by $\phi_{0}(z)$ up to exponentially small corrections. One has

$$
\begin{aligned}
I_{10}^{A} & =\int_{0}^{z_{0}} \mathrm{~d} z\left(Q_{N}^{2}(z)-\frac{1}{N^{2}}\right) \sim \int_{0}^{z_{0}} \mathrm{~d} z \phi_{0}^{2}(z)-\frac{z_{0}}{N^{2}} \\
& =\int_{0}^{1} \mathrm{~d} z \phi_{0}^{2}(z)+\int_{1}^{z_{0}} \mathrm{~d} z\left(\phi_{0}^{2}(z)-\frac{1}{4 \pi z}\right)+\frac{1}{4 \pi} \log z_{0}-\frac{z_{0}}{N^{2}} .
\end{aligned}
$$

In the second integral of (6.80) one can replace $S_{N}(x)$ by $S(x)$ up to O $\left(1 / N^{2}\right)$ correction.

$$
\begin{aligned}
I_{10}^{B}= & \frac{1}{4 \pi} \int_{x_{0}}^{\infty} \mathrm{d} x\left(S_{N}^{2}(x)-1\right) \sim \frac{1}{4 \pi} \int_{x_{0}}^{\infty} \mathrm{d} x\left(S^{2}(x)-1\right) \\
= & \frac{1}{4 \pi} \int_{x_{0}}^{1} \mathrm{~d} x\left(S^{2}(x)-\frac{1}{x}\right)+\frac{1}{4 \pi} \int_{1}^{\infty} \mathrm{d} x\left(S^{2}(x)-1\right) \\
& -\frac{1-x_{0}}{4 \pi}-\frac{1}{4 \pi} \log x_{0} .
\end{aligned}
$$


Adding the two terms and for large $N$ one obtains

$$
\begin{aligned}
I_{10}= & \int_{0}^{1} \mathrm{~d} z \phi_{0}^{2}(z)+\int_{0}^{\infty} \mathrm{d} z\left(\phi_{0}^{2}(z)-\frac{1}{4 \pi z}\right) \\
& +\frac{1}{4 \pi} \int_{0}^{1} \mathrm{~d} x\left(S^{2}(x)-\frac{1}{x}\right)+\frac{1}{4 \pi} \int_{1}^{\infty} \mathrm{d} x\left(S^{2}(x)-1\right) \\
& -\frac{1}{4 \pi}+\frac{1}{4 \pi} \log \left(\frac{N^{2}}{4 \pi}\right)+\mathrm{O}\left(1 / N^{2}\right) .
\end{aligned}
$$

Evaluating the integrals one has

$$
I_{10}=\frac{1}{2 \pi} \log N+0.048765633170141301742768467921+\mathrm{O}\left(1 / N^{2}\right) .
$$

For $\ell=1$ we trivially have

$$
I_{11}=\frac{1}{2} I_{00}=\frac{1}{2}-\frac{1}{2 N^{2}} .
$$

For $n \geq 2$ in the large $N$ limit the leading term of $I_{n 0}$ is proportional to $N^{2 n-2}$. The corresponding coefficient is given by (see [12])

$$
\lim _{N \rightarrow \infty} N^{2-2 n} I_{n 0}=\frac{1}{(2 \pi)^{2 n}} \sum_{k_{0}, k_{1}=-\infty}^{\infty}, \frac{1}{\left(\mathbf{k}^{2}\right)^{n}}=\frac{4}{(2 \pi)^{2 n}} \zeta(n) \beta(n),
$$

where $\zeta(n)$ and $\beta(n)$ are Riemann's zeta-function and Dirichlet's beta-function, respectively. In particular, up to $\mathrm{O}\left(N^{-2}\right)$ one has

$$
\frac{1}{V} I_{20}=\frac{K}{24 \pi^{2}}+\ldots=0.0038669465907372100307+\ldots
$$

where $K=0.91596559417721901505$ is Catalan's constant (since $\zeta(2)=\pi^{2} / 6$ and $\beta(2)=$ $K)$. One also has

$$
\frac{1}{V} I_{31}=\frac{1}{2 V} I_{20}=0.0019334732953686050153+\ldots
$$

Alternatively from the integral representation

$$
\frac{1}{V} I_{20}=\frac{1}{(4 \pi)^{2}} \int_{0}^{\infty} \mathrm{d} x x\left[S^{2}(x)-1\right]+\mathrm{O}\left(N^{-2}\right)
$$

the evaluation of which agrees to all digits with (6.87).

Further we have

$$
I_{21}=\frac{1}{2} I_{10}
$$

and $[9]$

$$
I_{22 ; 0}=\int_{0}^{\infty} \mathrm{d} z z \phi_{0}(z) \phi_{0}^{\prime \prime}(z)=\frac{1}{2}-\frac{1}{2 \pi} .
$$


Next

$$
\begin{aligned}
I_{32}= & \frac{1}{2} \int_{0}^{x_{0}} \mathrm{~d} z z^{2} \phi_{0}(z) \phi_{0}^{\prime \prime}(z)+\frac{1}{8 \pi} \int_{x_{0}}^{\infty} \mathrm{d} x x^{2} S(x) S^{\prime \prime}(x) \\
= & \frac{1}{2} \int_{0}^{1} \mathrm{~d} z z^{2} \phi_{0}(z) \phi_{0}^{\prime \prime}(z)+\frac{1}{2} \int_{1}^{\infty} \mathrm{d} z z^{2}\left(\phi_{0}(z) \phi_{0}^{\prime \prime}(z)-\frac{3}{16 \pi z^{3}}\right) \\
& +\frac{1}{8 \pi} \int_{0}^{1} \mathrm{~d} x x^{2}\left(S(x) S^{\prime \prime}(x)-\frac{3}{4 \pi x^{3}}\right) \\
& +\frac{1}{8 \pi} \int_{1}^{\infty} \mathrm{d} x x^{2} S(x) S^{\prime \prime}(x)+\frac{3}{32 \pi} \log \left(\frac{N^{2}}{4 \pi}\right) \\
= & \frac{3}{16 \pi} \log N+0.0212534753416951596 . \\
I_{33 ; 0}= & -\frac{1}{2} \int_{0}^{\infty} \mathrm{d} z z^{2} \phi_{0}(z) \phi_{0}^{(3)}(z)=\frac{1}{2}-\frac{3}{4 \pi} .
\end{aligned}
$$

\subsection{Some 2-loop momentum sums}

In this subsection we consider only the following 2-loop lattice sums which appear in the computations [1]:

$$
\begin{aligned}
& W_{3 a}=-\sum_{x} G(x)^{2} \nabla_{0}^{2} G(x), \\
& W_{3 c}=\sum_{x} \nabla_{0} G(x)\left[\left(\partial_{0} G(x)\right)^{2}-\left(\partial_{0}^{*} G(x)\right)^{2}\right]=-\frac{1}{6} \sum_{x}\left[\square_{0} G(x)\right]^{3} .
\end{aligned}
$$

It is sometimes useful to write

$$
W_{3 a}=\widetilde{W}_{3 a}-2 G(0) \sum_{x} G(x) \nabla_{0}^{2} G(x),
$$

where

$$
\widetilde{W}_{3 a}=-\sum_{x}[G(x)-G(0)]^{2} \nabla_{0}^{2} G(x)
$$

Using $\nabla_{0}^{2}=\square_{0}+\frac{1}{4} \square_{0}^{2}$ :

$$
\begin{aligned}
\sum_{x} G(x) \nabla_{0}^{2} G(x) & =-I_{21}+\frac{1}{4} I_{22}, \\
\widetilde{W}_{3 a} & =-\sum_{x}[G(x)-G(0)]^{2} \square_{0} G(x)+\frac{1}{2} A_{1},
\end{aligned}
$$

where

$$
A_{1}=-\frac{1}{2} \sum_{x}[G(x)-G(0)]^{2} \square_{0}^{2} G(x) .
$$

For the symmetric case $\ell=1$ we have

$$
W_{3 a}=I_{10}\left(\frac{1}{d} I_{10}-\frac{1}{2} I_{22}\right)-\frac{1}{d V} I_{20}+\frac{1}{2} A_{1}, \quad \ell=1 .
$$




\subsubsection{Case $d=2$}

In this case we only consider the symmetric case where we can use (6.101) and previous results to obtain

$$
W_{3 a}=\frac{1}{8 \pi^{2}} \ln ^{2} N+\frac{\ln N}{2 \pi}\left(I_{10 ; 0}-\frac{1}{4}+\frac{1}{4 \pi}\right)+W_{3 a ; 0}+\mathrm{O}\left(1 / N^{2}\right), \quad \ell=1,
$$

with

$$
W_{3 a ; 0}=\frac{1}{2} I_{10 ; 0}\left(I_{10 ; 0}-\frac{1}{2}+\frac{1}{2 \pi}\right)-\frac{K}{48 \pi^{2}}+\frac{1}{2} a_{1}, \quad \ell=1,
$$

where

$$
\begin{aligned}
a_{1} & =-\frac{1}{4} \int_{k, l} \frac{E_{k+l}-E_{k}-E_{l}}{E_{k} E_{l} E_{k+l}^{2}} \sum_{\mu}{\widehat{(k+l})_{\mu}^{4}}^{4} \\
& =0.0461636292439177762(1),
\end{aligned}
$$

where $E_{k}=\hat{k}^{2}$. Putting in the numerical value for $I_{10 ; 0}$ for $\ell=1$ we obtain

$$
W_{3 a ; 0}=0.0140266223093143915(1), \quad \ell=1 .
$$

For the other double sum

$$
W_{3 c}=W_{3 c ; 0}+\mathrm{O}\left(N^{-2}\right)
$$

where $W_{3 c ; 0}$ is the infinite volume sum which can be obtained for example by computing $W_{3 c}$ for $\ell=1$ :

$$
\begin{aligned}
-6 W_{3 c}(\ell=1) & =\frac{1}{2} \sum_{x}\left\{\left[\square_{0} G(x)\right]^{3}+\left[\square_{1} G(x)\right]^{3}\right\} \\
& =\frac{1}{2} \sum_{x}\left\{\square_{0} G(x)+\square_{1} G(x)\right\}\left\{\left[\square_{0} G(x)\right]^{2}-\square_{0} G(x) \square_{1} G(x)+\left[\square_{1} G(x)\right]^{2}\right\} \\
& =-\frac{1}{2} \sum_{x}\left\{\delta(x)-\frac{1}{V}\right\}\left\{\left[\square_{0} G(x)\right]^{2}-\square_{0} G(x) \square_{1} G(x)+\left[\square_{1} G(x)\right]^{2}\right\} \\
& =-\frac{1}{8}\left(1-\frac{1}{V}\right)^{2}+\frac{1}{2 V} \sum_{x}\left\{\left[\square_{0} G(x)\right]^{2}-\square_{0} G(x) \square_{1} G(x)+\left[\square_{1} G(x)\right]^{2}\right\} \\
& =-\frac{1}{8}+\mathrm{O}(1 / V) .
\end{aligned}
$$

So

$$
W_{3 c ; 0}=\frac{1}{48}, \quad d=2
$$

\subsubsection{Case $d=3$}

We have determined the expansions by fitting the sums for a range of values of $N$ for fixed $\ell$. For the leading terms we have

$$
\begin{aligned}
& W_{3 a ; 0}=0.007958105980, \\
& W_{3 c ; 0}=0.005702360998 .
\end{aligned}
$$


The large $N$ expansions are of the form:

$$
\begin{aligned}
& W_{3 a}[\ell=1]=W_{3 a ; 0}-0.019432034237 \frac{1}{N}+0.013457106339 \frac{1}{N^{2}}+\mathrm{O}\left(N^{-3}\right), \\
& W_{3 a}[\ell=2]=W_{3 a ; 0}-0.012367818370 \frac{1}{N}+0.012044486715 \frac{1}{N^{2}}+\mathrm{O}\left(N^{-3}\right),
\end{aligned}
$$

and

$$
W_{3 c}=W_{3 c ; 0}+\mathrm{O}\left(N^{-3}\right) \text {. }
$$

The coefficient $W_{3 a ; 2}$ is related to $\psi, \beta_{1}, \bar{\beta}_{1}$ defined in [11] through

$$
W_{3 a ; 2}=\psi+\frac{1}{3} \beta_{1} \bar{\beta}_{1} .
$$

\subsubsection{Case $d=4$}

Again we have obtained the expansions by fitting the sums for a range of $N$ for fixed $\ell$. For the infinite volume limit we obtained

$$
\begin{aligned}
& W_{3 a ; 0}=0.001850792346021407306 \\
& W_{3 c ; 0}=0.00227599909081849438
\end{aligned}
$$

The expansion of $W_{3 a}$ is given by

$$
\begin{aligned}
& W_{3 a}[\ell=1]=W_{3 a ; 0}-0.004204473492568 N^{-2} \\
& -\left(32 \pi^{2}\right)^{-1} N^{-4} \ln N+0.0077108092 N^{-4}+\ldots \\
& W_{3 a}[\ell=2]=W_{3 a ; 0}-0.0017695104622235 N^{-2} \\
& +0.0081775349546087202407 N^{-4} \ln N+0.004168882181 N^{-4}+\ldots \\
& W_{3 a}[\ell=3]=W_{3 a ; 0}+0.00072483841 N^{-2} \\
& +0.011346635653943 N^{-4} \ln N+0.0131674 N^{-4}+\ldots \\
& W_{3 a}[\ell=4]=W_{3 a ; 0}+0.0032192873 N^{-2} \\
& +0.01292978432706041335 N^{-4} \ln N+0.033262 N^{-4}+\ldots
\end{aligned}
$$

The coefficient of $N^{-4} \ln N$ can be obtained from the DR calculation. This gives

$$
W_{3 a ; 4 x}=\frac{1}{48 \pi^{2}}\left(\frac{1}{\ell}-10 \ddot{g}(0 ; \ell)\right) .
$$

The fitting procedure reproduces this value.

For $W_{3 c}$ we obtain:

$$
\begin{aligned}
& W_{3 c}[\ell=1]=W_{3 c ; 0}-0.01188332621 N^{-4}+\ldots \\
& W_{3 c}[\ell=2]=W_{3 c ; 0}+0.01603789713 N^{-4}+\ldots \\
& W_{3 c}[\ell=3]=W_{3 c ; 0}+0.02396645060 N^{-4}+\ldots \\
& W_{3 c}[\ell=4]=W_{3 c ; 0}+0.027927570 N^{-4}+\ldots
\end{aligned}
$$


The computation in [1] required the following relations for consistency:

$$
\begin{aligned}
W_{3 a ; 2} & =\frac{1}{8}\left(8 I_{10 ; 0}-1\right) I_{10 ; 2}, \\
W_{3 a ; 4 x} & =\frac{1}{48 \pi^{2}}\left(10 I_{11 ; 4}+\frac{1}{\ell}\right), \\
W_{3 c ; 4} & =-\frac{1}{8}\left(4 I_{10 ; 0}-1\right) I_{11 ; 4} .
\end{aligned}
$$

These are satisfied by the numerical values above.

\section{Acknowledgments}

We like to thank Christoph Weiermann for collaboration at an early stage of the work. We would also like to thank Janos Balog, Peter Hasenfratz, Heiri Leutwyler and Martin Lüscher for useful discussions.

\section{A Derivation of formulae for $g(0)$ and $\ddot{g}(0)$ up to $O(D-4)$}

Using (3.56), (2.22) and (2.28) we have

$$
g(0) L^{D-2}=\frac{1}{4 \pi \mathcal{V}_{D}} \int_{0}^{\infty} \mathrm{d} u\left[\mathcal{S}_{D}\left(\frac{u}{\ell^{2}}\right)\right]_{\mathrm{sub}}-\frac{1}{4 \pi \mathcal{V}_{D}}-\frac{1}{2 \pi(D-2)} .
$$

Expanding in $q$ one gets (3.59) and (3.60).

To calculate the second derivatives we use the relations

$$
\begin{aligned}
\left.\frac{\partial^{2}}{\partial z^{2}} S(v, z)\right|_{z=0} & =\sum_{n=-\infty}^{\infty}\left(4 \pi^{2} v^{2} n^{2}-2 \pi v\right) \mathrm{e}^{-\pi v n^{2}}=-2 \pi v(2 v T(v)+1) S(v), \\
\left.\frac{\partial^{2}}{\partial x_{\nu}^{2}} S\left(\frac{\ell_{\nu}^{2}}{u}, \frac{x_{\nu}}{L_{\nu}}\right)\right|_{x_{\nu}=0} & =-\frac{2 \pi l_{\nu}^{2}}{L^{2} u}\left[\frac{2 \ell_{\nu}^{2}}{u} T\left(\frac{\ell_{\nu}^{2}}{u}\right)+1\right] S\left(\frac{\ell_{\nu}^{2}}{u}\right) .
\end{aligned}
$$

From (3.55) one obtains then

$$
\begin{aligned}
\partial_{\nu}^{2} g(0) L^{D} & =-\frac{1}{2} \int_{0}^{\infty} \frac{\mathrm{d} u}{u^{D / 2+1}}\left\{\left[\frac{2 \ell_{\nu}^{2}}{u} T\left(\frac{\ell_{\nu}^{2}}{u}\right)+1\right] \mathcal{S}_{D}\left(\frac{\ell^{2}}{u}\right)-1\right\} \\
& =\int_{0}^{\infty} \mathrm{d} u\left\{\frac{1}{\ell_{\nu}^{2} \mathcal{V}_{D}} T\left(\frac{u}{\ell_{\nu}^{2}}\right) \mathcal{S}_{D}\left(\frac{u}{\ell^{2}}\right)+\frac{1}{2 u^{D / 2+1}}\right\} \\
& =\frac{1}{\ell_{\nu}^{2} \mathcal{V}_{D}} \int_{0}^{\infty} \mathrm{d} u\left[T\left(\frac{u}{\ell_{\nu}^{2}}\right) \mathcal{S}_{D}\left(\frac{u}{\ell^{2}}\right)\right]_{\mathrm{sub}}+\frac{1}{D}
\end{aligned}
$$

Expanding in $q$ one gets (3.61) and (3.62).

\section{B Evaluation of $\Psi$ for the massive case in DR}

Here we first considered the integral over the torus $\mathcal{T}=\prod_{\mu=0}^{d-1} S^{1}\left(L_{\mu}\right)\left[S^{1}(\widehat{L})\right]^{q}$ :

$$
\bar{W}(M)=-\int_{x \in \mathcal{T}}[G(x, M)]^{2} \partial_{\nu}^{2} G(x, M), \text { no sum over } \nu,
$$

with the massive free propagator $G(x, M)$. 
Using the Schwinger representation:

$$
\begin{gathered}
\bar{W}(M)=\int_{x \in \mathcal{T}}\left[\prod_{j=1}^{3} \int_{0}^{\infty} \mathrm{d} t_{j}\right] \mathcal{W}, \\
\mathcal{W}=\mathrm{e}^{-M^{2}\left(t_{1}+t_{2}+t_{3}\right)} \frac{1}{V_{D}} \sum_{p_{1}} p_{1 \nu}^{2} \exp \left\{i p_{1} x-p_{1}^{2} t_{1}\right\} \prod_{k=2}^{3}\left(\frac{1}{V_{D}} \sum_{p_{k}} \exp \left\{i p_{k} x-p_{k}^{2} t_{k}\right\}\right) .
\end{gathered}
$$

We then break the $t_{k}$ integrations into parts $\left(t_{0}=L^{2} /(4 \pi)\right)$ :

$$
\bar{W}=\bar{W}^{(1)}+2 \bar{W}^{(2)}+\bar{W}^{(3)}+\bar{W}^{(4)}+2 \bar{W}^{(5)}+\bar{W}^{(6)},
$$

with

$$
\begin{aligned}
& \bar{W}^{(1)}=\int_{x \in \mathcal{T}} \int_{t_{0}}^{\infty} \mathrm{d} t_{1} \int_{t_{0}}^{\infty} \mathrm{d} t_{2} \int_{t_{0}}^{\infty} \mathrm{d} t_{3} \mathcal{W}, \\
& \bar{W}^{(2)}=\int_{x \in \mathcal{T}} \int_{t_{0}}^{\infty} \mathrm{d} t_{1} \int_{t_{0}}^{\infty} \mathrm{d} t_{2} \int_{0}^{t_{0}} \mathrm{~d} t_{3} \mathcal{W}, \\
& \bar{W}^{(3)}=\int_{x \in \mathcal{T}} \int_{0}^{t_{0}} \mathrm{~d} t_{1} \int_{t_{0}}^{\infty} \mathrm{d} t_{2} \int_{t_{0}}^{\infty} \mathrm{d} t_{3} \mathcal{W}, \\
& \bar{W}^{(4)}=\int_{x \in \mathcal{T}} \int_{t_{0}}^{\infty} \mathrm{d} t_{1} \int_{0}^{t_{0}} \mathrm{~d} t_{2} \int_{0}^{t_{0}} \mathrm{~d} t_{3} \mathcal{W}, \\
& \bar{W}^{(5)}=\int_{x \in \mathcal{T}} \int_{0}^{t_{0}} \mathrm{~d} t_{1} \int_{0}^{t_{0}} \mathrm{~d} t_{2} \int_{t_{0}}^{\infty} \mathrm{d} t_{3} \mathcal{W}, \\
& \bar{W}^{(6)}=\int_{x \in \mathcal{T}} \int_{0}^{t_{0}} \mathrm{~d} t_{1} \int_{0}^{t_{0}} \mathrm{~d} t_{2} \int_{0}^{t_{0}} \mathrm{~d} t_{3} \mathcal{W},
\end{aligned}
$$

and considered these separately. The advantage of this procedure is that in each case the $x$ integration can be done first analytically. The disadvantage over the coordinate space method is that there are many terms to be considered and the integration over the $t_{i}$ is often quite difficult.

The term $\bar{W}^{(1)}$ is the simplest:

$$
\begin{aligned}
\bar{W}^{(1)}(M)= & \frac{1}{V_{D}^{2}}\left[\prod_{j=1}^{3} \int_{t_{0}}^{\infty} \mathrm{d} t_{j}\right] \mathrm{e}^{-M^{2}\left(t_{1}+t_{2}+t_{3}\right)} \sum_{p_{1}, p_{2}} p_{1 \nu}^{2} \exp \left\{-p_{1}^{2} t_{1}-p_{2}^{2} t_{2}-\left(p_{1}+p_{2}\right)^{2} t_{3}\right\} \\
= & \frac{1}{V_{D}^{2}}\left(\frac{L^{2}}{4 \pi}\right)^{3} \frac{4 \pi^{2}}{L_{\nu}^{2}}\left[\prod_{j=1}^{3} \int_{1}^{\infty} \mathrm{d} t_{j}\right] \mathrm{e}^{-\left(t_{1}+t_{2}+t_{3}\right) z^{2} /(4 \pi)} \\
& \times \sum_{n_{1}, n_{2}} n_{1 \nu}^{2} \exp \left\{-\sum_{\mu} \frac{\pi}{\ell_{\mu}^{2}}\left[n_{1 \mu}^{2} t_{1}+n_{2 \mu}^{2} t_{2}+\left(n_{1 \mu}+n_{2 \mu}\right)^{2} t_{3}\right]\right\} \\
= & \frac{L^{4-2 D}}{16 \pi \mathcal{V}^{2} \hat{\ell}^{2 q} \ell_{\nu}^{2}} \sum_{m, n} m_{\nu}^{2} R(z, m / \ell) R(z, n / \ell) R(z,(m+n) / \ell)
\end{aligned}
$$


where $z=M L$ and

$$
\begin{aligned}
& R(z, v) \equiv \frac{\exp \{-r(z, v)\}}{r(z, v)}, \\
& r(z, v) \equiv \frac{z^{2}}{4 \pi}+\pi v^{2} .
\end{aligned}
$$

Splitting off the terms in the double sum with $n=0$ and $n+m=0$ we can separate the singularities as $M \rightarrow 0$ and the remaining sums can be computed accurately.

Next we turn to $\bar{W}^{(2)}$; using the Poisson summation formula (3.49) we obtain

$$
\begin{aligned}
\bar{W}^{(2)}(M)= & \frac{1}{V_{D}^{2}} \int_{x \in \mathcal{T}} \int_{t_{0}}^{\infty} \mathrm{d} t_{1} \int_{t_{0}}^{\infty} \mathrm{d} t_{2} \int_{0}^{t_{0}} \mathrm{~d} t_{3} \mathrm{e}^{-M^{2}\left(t_{1}+t_{2}+t_{3}\right)} \\
& \times\left(4 \pi t_{3}\right)^{-D / 2} \sum_{n} \exp \left(-\frac{\sum_{\mu}\left(x_{\mu}+n_{\mu} L_{\mu}\right)^{2}}{4 t_{3}}\right) \\
& \times \sum_{p_{1}} p_{1 \nu}^{2} \exp \left\{i p_{1} x-p_{1}^{2} t_{1}\right\} \sum_{p_{2}} \exp \left\{i p_{2} x-p_{2}^{2} t_{2}\right\} .
\end{aligned}
$$

An important step now, which is also used for the remaining $\bar{W}^{(a)}$, is to use translation invariance to replace the $x$ integration over $\mathcal{T}$ by an integration over $\mathbb{R}^{D}$ which is readily performed:

$$
\begin{aligned}
\bar{W}^{(2)}(M)= & \frac{1}{V_{D}^{2}} \int_{x \in \mathbb{R}^{D}} \int_{t_{0}}^{\infty} \mathrm{d} t_{1} \int_{t_{0}}^{\infty} \mathrm{d} t_{2} \int_{0}^{t_{0}} \mathrm{~d} t_{3} \mathrm{e}^{-M^{2}\left(t_{1}+t_{2}+t_{3}\right)}\left(4 \pi t_{3}\right)^{-D / 2} \exp \left(-\frac{x^{2}}{4 t_{3}}\right) \\
& \times \sum_{p_{1}} p_{1 \nu}^{2} \exp \left\{i p_{1} x-p_{1}^{2} t_{1}\right\} \sum_{p_{2}} \exp \left\{i p_{2} x-p_{2}^{2} t_{2}\right\} \\
= & \frac{1}{V_{D}^{2}} \int_{t_{0}}^{\infty} \mathrm{d} t_{1} \int_{t_{0}}^{\infty} \mathrm{d} t_{2} \int_{0}^{t_{0}} \mathrm{~d} t_{3} \mathrm{e}^{-M^{2}\left(t_{1}+t_{2}+t_{3}\right)} \\
& \times \sum_{p_{1}, p_{2}} p_{1 \nu}^{2} \exp \left\{-p_{1}^{2} t_{1}-p_{2}^{2} t_{2}-\left(p_{1}+p_{2}\right)^{2} t_{3}\right\} \\
= & \frac{1}{V_{D}^{2}}\left(\frac{L^{2}}{4 \pi}\right)^{3} \frac{4 \pi^{2}}{L_{\nu}^{2}} \int_{1}^{\infty} \mathrm{d} t_{1} \int_{1}^{\infty} \mathrm{d} t_{2} \int_{0}^{1} \mathrm{~d} t_{3} \mathrm{e}^{-\left(t_{1}+t_{2}+t_{3}\right) z^{2} /(4 \pi)} \\
& \times \sum_{n_{1}, n_{2}} n_{1 \nu}^{2} \exp \left\{-\sum_{\mu} \frac{\pi}{\ell_{\mu}^{2}}\left[n_{1 \mu}^{2} t_{1}+n_{2 \mu}^{2} t_{2}+\left(n_{1}+n_{2}\right)_{\mu}^{2} t_{3}\right]\right\} \\
= & \frac{L^{4-2 D}}{16 \pi \mathcal{V}^{2} \hat{\ell}^{2 q} \ell_{\nu}^{2}} \sum_{m, n} m_{\nu}^{2} R(z, m / \ell) R(z, n / \ell) \bar{R}(z,(m+n) / \ell)
\end{aligned}
$$

where

$$
\bar{R}(z, v) \equiv \frac{1-\exp \{-r(z, v)\}}{r(z, v)}
$$

The computation of all terms is too lengthy to present here. The terms $\bar{W}^{(4)}, \bar{W}^{(5)}$, $\bar{W}^{(6)}$ have poles at $D=4$ which are separated analytically. The remaining integrals over the $t_{i}$ continued to $D=4$ are computable using e.g. NAGLIB routines.

Note that the singularities as $M \rightarrow 0$ in $\bar{W}(M)$ come from the terms $p=0$ in the propagators at finite volume. Our finite volume zero mass propagators exclude the zero mode. 


\section{Some useful relations involving the lattice propagator}

Some other useful relations are:

$$
\begin{aligned}
G(\hat{0}) & =I_{10}-\frac{1}{2} I_{11}, \\
G(\hat{k}) & =I_{10}+\frac{1}{2 d_{s}}\left(I_{11}-I_{00}\right), \\
\partial_{0} G(0) & =-\frac{1}{2} I_{11}, \\
\partial_{0}^{*} G(0) & =\frac{1}{2} I_{11}, \\
\partial_{k} G(0) & =-\frac{1}{2 d_{s}}\left(I_{00}-I_{11}\right), \\
\partial_{k}^{*} G(0) & =\frac{1}{2 d_{s}}\left(I_{00}-I_{11}\right), \\
\square_{0} G(0) & =-I_{11}, \\
\square G(0) & =-I_{00}, \\
\partial_{0}^{2} G(0) & =-I_{11}+\frac{1}{2} I_{12} \\
\partial_{k}^{2} G(0) & =\frac{1}{d_{s}}\left(-I_{00}+I_{11}-\frac{1}{2} I_{12}+\frac{1}{2} J_{10}\right), \\
\partial_{0}^{*} \partial_{k} G(0) & =-\frac{1}{4 d_{s}}\left(I_{01}-I_{12}\right), \\
\partial_{0} \square \square_{0} G(0) & =\frac{1}{2} I_{12}, \\
\partial_{0}^{*} \partial_{k}^{2} G(0) & =\frac{1}{2 d_{s}}\left(-I_{01}+I_{12}-\frac{1}{2} I_{13}+\frac{1}{2} J_{11}\right), \\
\square \square_{0}^{*} G(0) & =I_{12}, \\
\square \partial_{0}^{*} G(0) & =I_{01}^{2} G(0)=\frac{1}{d_{s}}\left(I_{01}-I_{12}-\frac{1}{2} I_{02}+I_{13}-\frac{1}{4} I_{14}-\frac{1}{2} J_{11}+\frac{1}{4} J_{12}\right) .
\end{aligned}
$$

\section{Modified Bessel function}

For integer order $n$ the modified Bessel function can be calculated by

$$
I_{n}(x)=\sum_{k=0}^{\infty} \frac{1}{k !(n+k) !}\left(\frac{x}{2}\right)^{2 k+n} .
$$

For $n \gg x$ one has

$$
I_{n}(x) \sim \frac{1}{n !}\left(\frac{x}{2}\right)^{n} \sim \frac{1}{\sqrt{2 \pi n}}\left(\frac{\mathrm{e} x}{2 n}\right)^{n} .
$$

For $\nu=$ fixed, $x \rightarrow \infty$ :

$$
\mathrm{e}^{-x} I_{\nu}(x) \sim \frac{1}{\sqrt{2 \pi x}} \sum_{k=0}^{\infty}(-1)^{k} \frac{a_{k}(\nu)}{x^{k}}
$$


where

$$
a_{k}(\nu)=\frac{\left(4 \nu^{2}-1\right)\left(4 \nu^{2}-3^{2}\right) \ldots\left(4 \nu^{2}-(2 k-1)^{2}\right)}{k ! 8^{k}} .
$$

For $n=0$ one has

$$
I_{0}(x)=\sum_{k=0}^{\infty} \frac{1}{(k !)^{2}}\left(\frac{x}{2}\right)^{2 k}
$$

and for $x \rightarrow \infty$

$$
\begin{aligned}
\mathrm{e}^{-x} I_{0}(x) & \sim \frac{1}{\sqrt{2 \pi x}} \sum_{k=0}^{\infty}\left(\frac{(2 k) !}{2^{k}(k !)^{2}}\right)^{2} \frac{1}{k !(8 x)^{k}} \\
& =\frac{1}{\sqrt{2 \pi x}}\left(1+\frac{1}{8 x}+\frac{9}{128 x^{2}}+\ldots\right) .
\end{aligned}
$$

A uniform expansion for $I_{\nu}(\nu x)$ is $\nu \rightarrow \infty$

$$
\mathrm{e}^{-\nu x} I_{\nu}(\nu x) \sim \frac{\mathrm{e}^{\nu \rho}}{\sqrt{2 \pi \nu}\left(1+x^{2}\right)^{1 / 4}} \sum_{k=0}^{\infty} \frac{U_{k}(p)}{\nu^{k}},
$$

where

$$
\begin{aligned}
U_{0}(p) & =1 \\
U_{1}(p) & =\frac{1}{24}\left(3 p-5 p^{3}\right) \\
U_{2}(p) & =\frac{1}{1152}\left(81 p^{2}-462 p^{4}+385 p^{6}\right),
\end{aligned}
$$

and

$$
\begin{aligned}
& \rho=\sqrt{1+x^{2}}-x+\ln \left(\frac{x}{1+\sqrt{1+x^{2}}}\right), \\
& p=\left(1+x^{2}\right)^{-1 / 4} .
\end{aligned}
$$

Eq. (D.7) can also be regarded as asymptotic expansion for $x \rightarrow \infty, \nu=$ fixed.

For large $x$ one has $\rho=-1 /(2 x)+\mathrm{O}\left(x^{-3}\right)$. This shows that for $x_{n} \lesssim n^{\alpha}$ with $\alpha<2$ for $n \rightarrow \infty \mathrm{e}^{-x_{n}} I_{n}\left(x_{n}\right)$ decreases exponentially fast. Therefore

$$
N^{\alpha / 2}\left[Q_{N}\left(x N^{\alpha}\right)-\phi_{0}\left(x N^{\alpha}\right)\right]=\mathrm{O}\left(\exp \left(-N^{2-\alpha} / x\right)\right), \quad 0 \leq \alpha<2 .
$$

Using (D.6) one obtains in the large $N$ limit a simple scaling function,

$$
\lim _{N \rightarrow \infty} N^{\alpha / 2} Q_{N}\left(x N^{\alpha}\right)=\frac{1}{\sqrt{4 \pi x}}, \quad 0<\alpha<2 .
$$

For $\alpha=2$ this is not true any more, and according to (6.29) one obtains a non-trivial scaling function

$$
\lim _{N \rightarrow \infty} N Q_{N}\left(x N^{2}\right)=S(4 \pi x) .
$$

Summarizing one has three different scaling regimes

$$
\lim _{N \rightarrow \infty} N^{\alpha / 2} Q_{N}\left(x N^{\alpha}\right)= \begin{cases}\phi_{0}(x), & \alpha=0, \\ 1 / \sqrt{4 \pi x} & 0<\alpha<2, \\ S(4 \pi x), & \alpha=2 .\end{cases}
$$


Open Access. This article is distributed under the terms of the Creative Commons Attribution License (CC-BY 4.0), which permits any use, distribution and reproduction in any medium, provided the original author(s) and source are credited.

\section{References}

[1] F. Niedermayer and P. Weisz, Matching effective chiral Lagrangians with dimensional and lattice regularizations, JHEP 04 (2016) 110 [arXiv:1601.00614] [INSPIRE].

[2] P. Hasenfratz and H. Leutwyler, Goldstone Boson Related Finite Size Effects in Field Theory and Critical Phenomena With O(N) Symmetry, Nucl. Phys. B 343 (1990) 241 [InSPIRE].

[3] J. Bijnens, Sunset integrals at finite volume, PoS (LATTICE 2013) 112 [arXiv:1310.0350] [INSPIRE].

[4] J. Bijnens, E. Boström and T.A. Lähde, Two-loop Sunset Integrals at Finite Volume, JHEP 01 (2014) 019 [arXiv:1311.3531] [InSPIRE].

[5] M. Lüscher, P. Weisz and U. Wolff, A numerical method to compute the running coupling in asymptotically free theories, Nucl. Phys. B 359 (1991) 221 [INSPIRE].

[6] P. Hasenfratz, The QCD rotator in the chiral limit, Nucl. Phys. B 828 (2010) 201 [arXiv: 0909.3419] [INSPIRE].

[7] M. Lüscher and P. Weisz, Efficient Numerical Techniques for Perturbative Lattice Gauge Theory Computations, Nucl. Phys. B 266 (1986) 309 [INSPIRE].

[8] M. Lüscher and P. Weisz, Coordinate space methods for the evaluation of Feynman diagrams in lattice field theories, Nucl. Phys. B 445 (1995) 429 [hep-lat/9502017] [INSPIRE].

[9] D.-S. Shin, Application of a coordinate space method for the evaluation of lattice Feynman diagrams in two-dimensions, Nucl. Phys. B 525 (1998) 457 [hep-lat/9706014] [InSPIRE].

[10] S. Necco and R. Sommer, The $N(f)=0$ heavy quark potential from short to intermediate distances, Nucl. Phys. B 622 (2002) 328 [hep-lat/0108008] [INSPIRE].

[11] P. Hasenfratz and F. Niedermayer, Finite size and temperature effects in the AF Heisenberg model, Z. Phys. B 92 (1993) 91 [hep-lat/9212022] [INSPIRE].

[12] J.M. Borwein and P.B. Borwein, Pi and the AGM: a study in analytic number theory and computational complexity, Wiley, New York, U.S.A. (1987). 